\title{
High-Strouhal-number pulsatory flow in a curved pipe
}

\author{
Feroz Ahmed ${ }^{1} \dagger$, Ian Eames ${ }^{1}$, Emad Moeendarbary $^{2}$ and Alireza \\ Azarbadegan ${ }^{3}$
}

\begin{abstract}
${ }^{1}$ Engineering in Extreme Environments Group (E-cubed), Department of Mechanical Engineering, University College London, Torrington Place, London, WC1E 7JE, UK

${ }^{2}$ Mechanics in Biology \& Medicine Group (MecBioMed), Department of Mechanical Engineering, University College London, Torrington Place, London, WC1E 7JE, UK

${ }^{3}$ BP Exploration Operating Company Limited, Chertsey Road, Sunbury-on-Thames, Middlesex, TW16 7NL, UK
\end{abstract}

(Received xx; revised xx; accepted xx)

The high-Strouhal-number pulsatory flow in a curved pipe is studied numerically. A general force analysis is developed for the bend force, where the new contribution from flow acceleration is identified and analysed. The mechanisms of secondary flow production are studied by extending Hawthorne's (1951) model to account for viscous effects and applied to assess the distinct contributions from stretching and no-slip condition. A detailed comparison is made between the numerical simulations and models for a pipe flow characterised by a volume flux $Q=U_{b} A\left|\sin \Omega_{p} t\right|\left(U_{b}\right.$ is maximum velocity and $\Omega_{p}$ is angular frequency). For high-Reynolds-number $\left(R e_{b}\right)$ and high-Strouhal-numbers $(S t)$, the bend force predictions are in good agreement with the numerical results over a wide range of bend curvature $\left(R_{c} / D\right.$; where $R_{c}$ is bend radius of curvature and $D$ is pipe diameter) owing to the influence of the streamwise flow acceleration on the pressure field. At high-St, the linear model shows that the source of streamwise vorticity of secondary flow by inviscid stretching is comparable to the component generated by viscous effects.

Key words: pulsatory flow, secondary flow, impulsive loading

\section{Introduction}

Unsteady flows along circular pipes are among the most studied fluid mechanics problems, encompassing a type of geometry (the circular tube) and a type of flow driven by a heart (Rindt et al. 1991; Najjari \& Plesniak 2016) or a pump (Singh \& Madavan 1987; Miller 1988; Manring 2000) common in nature and industry. Unsteady pipe flows in this context are broadly distinguished based on whether the flow at some stage reverses (Sudo et al. 1992) or remains positive (Singh \& Madavan 1987; Miller 1987; Vetter \& Schweinfurther 1987; Miller 1988; Sumida et al. 1989; Manring 2000; Takeshi et al. 1990; Komai \& Tanishita 1997; Krishna et al. 2017). The combination of flow unsteadiness and piping geometrical discontinuity (curved pipes) leads to large unsteady forces, which is problematic with respect to the piping stress and vibration. In the energy sector (oil and gas industry), the consequence of excessive pipeline stresses and vibration is the loss

$\dagger$ Email address for correspondence: ucemfah@ucl.ac.uk 


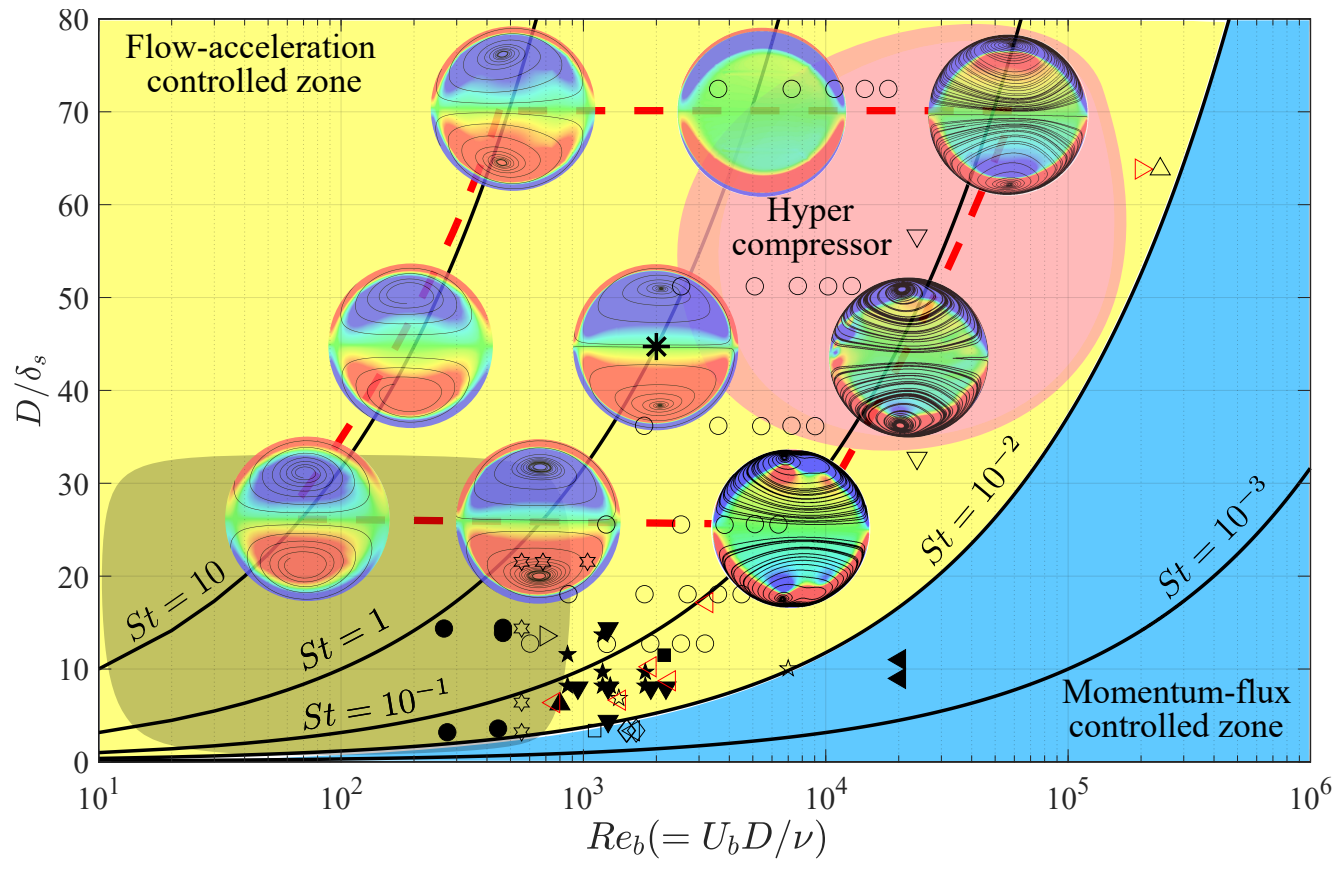

Figure 1. Regime diagram showing a summary of studies performed on unsteady pipe bend flow where the relative size of the pipe to oscillatory boundary layer thickness $\left(D / \delta_{s}\right)$ is plotted against maximum bulk Reynolds number $\left(R e_{b}\right)(2.3)$. Isocontours of Strouhal number $(S t)$ (2.5) are plotted for $S t=10^{-3}, 10^{-2}, 10^{-1}, 1$ and 10 . The empty and filled markers correspond to oscillatory and pulsatory flows, respectively (see table 1). The Grey patch region shows the regime plot range of Sudo et al. (1992). The approximate operating region for an industrial hyper-compressor is shown. The red-dashed boundary encloses the computations performed in this paper.

in structural integrity (for example, fatigue failures, instrument distortion, and process efficiency) and production downtime.

The purpose of this paper is to understand the influence of a pulsatory flow on pipe bend force and secondary flow when the Strouhal number is high and flow acceleration is important. This is illustrated with the case of volume flux $Q=U_{b} A\left|\sin \Omega_{p} t\right|$, which is typical of the idealised flow generated by high-energy reciprocating pumps. This type of flow has a rms comparable to the mean, so both (mean) steady and the oscillatory components are essential for the secondary flow and is characterised by a discontinuity in the flow acceleration. The general analysis developed will be relevant to a broader range of high-St inertially-dominated pulsatory flows irrespective of the nature of volume flux.

We discuss the main contributions to the secondary flow analysis first. The motivation for examining steady flows in curved pipes has been driven mainly by observing a weak steady secondary flow that remains persistent after a pipe bend. The commonly observed meandering of rivers has its origin in the secondary flow, which causes sediment accumulation on the inside bend and erosion on the outer bend (Thomson 1877). Einstein (1926) also noted the influence of secondary flows in the accumulation of tea leaves swept into the center of a cup after stirring has ceased. The enhanced shear stress induced by a secondary flow also has implications for hemodynamics in cardiovascular systems (Siggers \& Waters 2008; Cox et al. 2019), hence the vast body of research associated with this 


\begin{tabular}{|c|c|c|c|c|c|c|}
\hline & Authors & Analysis & Bend & Cross-section & $R_{c} / D$ & Flows \\
\hline • & Takeshi et al. (1990) & Exp. & $180^{\circ}$ & Circular & $1,3.5$ & Oscillatory \\
\hline$\Delta$ & Rindt et al. (1991) & Exp./Num. & $90^{\circ}$ & Circular & 3 & Mean oscillatory \\
\hline $\bar{\nabla}$ & Sumida (2007) & Exp. & $540^{\circ}$ & Circular & 5,15 & Mean oscillatory \\
\hline 4 & Hellstrom \& Fuchs (2007) & Num. & $90^{\circ}$ & Circular & $1,1.5$ & Mean oscillatory \\
\hline$\star$ & Timite et al. (2010) & Exp./Num. & $90^{0}$ & Circular & 5.5 & Mean oscillatory \\
\hline 23 & Komai \& Tanishita (1997) & Num. & $180^{\circ}$ & Circular & $1,1.5,3.5$ & Intermittent \\
\hline 类 & Mahmoudi Zarandi (2000) & Exp. & $180^{\circ}$ & Circular & 1.85 & Pulsatile \\
\hline$\triangleleft$ & Boiron et al. (2007) & Exp./Num. & $180^{\circ}$ & Circular & 6.82 & Intermittent \\
\hline$\triangleright$ & Kalpakli et al. (2010) & Exp. & $90^{\circ}$ & Circular & 0.84 & Pulsatile \\
\hline$\nabla$ & Vester et al. (2013) & Exp. & $90^{\circ}$ & Circular & 1.23 & Pulsatile \\
\hline$\triangle$ & Vester et al. (2015) & Exp. & $90^{\circ}$ & Circular & 1.25 & Pulsatile \\
\hline$\diamond$ & Plesniak \& Bulusu (2016) & Exp. & $180^{\circ}$ & Circular & 3.50 & Pulsatile \\
\hline$\square$ & Najjari \& Plesniak (2016) & Exp. & $180^{\circ}$ & Circular & 3.50 & Pulsatile \\
\hline 0 & Krishna et al. (2017) & Exp./Num. & $180^{\circ}$ & Square & 1.67 & Single +ve pulse \\
\hline$\triangleleft$ & Najjari \& Plesniak (2018) & Exp. & $180^{\circ}$ & Circular & 3.50 & Pulsatile \\
\hline$\triangleright$ & Najjari et al. (2019) & Exp./Num. & $180^{\circ}$ & Circular & 3.50 & Oscillatory \\
\hline 米 & Present study & Num. & $90^{\circ}$ & Circular & 1.50 & Pulsatory \\
\hline
\end{tabular}

Table 1. Summary of studies on unsteady flows in curved pipes (see figure 1).

area. The mixing in the heat exchanger and particle erosion/deposition (Inthavong 2019) in a curved pipe due to secondary flow has a substantial impact on power loss in the industrial piping system.

There are a startlingly large number of papers on secondary flows due to the wide parameter space spanned by different types of geometry (pipe cross-section and bend curvature), entrance flow (parabolic or top-hat profile entry-flow), and flow-history (steady, oscillatory or pulsatory) (see figure 1 and table 1). The first theoretical analysis of secondary flows in curved pipes for a steady flow was formulated by Dean $(1927,1928)$, who developed an asymptotic analysis for viscously-dominated secondary flow (which is unchanged in character along the pipe) around an infinitely long curved pipe $R_{c} / D \gg 1$. When $R e_{b} / \sqrt{R_{c} / D} \ll 1$, the secondary flow is characterised by a counter-rotating dipole vortical arrangement (Dean vortices) whose speed scales as $R e_{b} D / R_{c}$. This is due to the diffraction and distortion of the primary mean flow (in the upstream pipe), where the imbalance in the centrifugal force and pressure gradient in the radial direction causes an outward-centrifuging of the central-core flow and an azimuthal motion of sidewall flow. Hawthorne (1951) showed that a secondary flow could be established inviscidly and developed a theoretical analysis for the rotation of isobaric surfaces in bends. The secondary flow grew with angular displacement along the pipe bend, ultimately oscillating, and Hawthorne (1951) confirmed his general predictions with a series of experiments. Hawthorne's (1951) inviscid analysis of vorticity creation through stretching has been further developed and applied to considerable effect by Lighthill (1956) and Hunt (1973). Hawthorne's (1951) technique has formed the basis of many turbulence studies and is now usually referred to as rapid-distortion theory (RDT). Hawthorne's (1951) approach is further developed in this paper with the inclusion of viscous effects.

When the pipe flow is unsteady, the topology of vorticity and streamlines in secondary flow can dramatically change. The difference in the secondary flow patterns between steady flows (dipole vortical arrangement) and oscillatory flows (quadrupole vortical 
arrangement) was first analysed by Lyne (1971) for laminar flow with high $D / \delta_{s}$ in a small-curvature pipe $\left(R_{c} / D \gg 1\right)$. The asymptotic analysis of Lyne (1971) showed a quadrupole vortical arrangement comprised of a thin wall-bounded motion driving flow from the inner to the outer bend (and back along the sidewall) and a weak core motion driving a stream from outside to the inside bend. Bertelsen (1975) confirmed the quadrupole cross-stream flow structure from experimental observations of the streamline pattern and measurements of the velocity profiles in a $180^{\circ}$ curved pipe. Sudo et al. (1992) undertook a parametric analysis of the influence of $R e_{b}$ and $D / \delta_{s}$ (see the range in figure 1) on the streamline patterns in a fixed geometry $\left(R_{c} / D=3.8\right)$. With the increase in $D / \delta_{s}$, the secondary flow patterns undergo a transition from Dean's dipole (at low driving frequency $D / \delta_{s} \gtrsim 1$ ) to Lyne's quadrupole (at $D / \delta_{s} \gg 1$ ) via deformed Dean, intermediate Dean-Lyne, and deformed Lyne vortical arrangement (Sudo et al. 1992).

In general, a pipe flow may contain a mean (steady) and oscillatory component (called pulsatory flow), which together generates a secondary flow having the features of both Dean (1928) and Lyne (1971). Two types of pulsatory flows extensively studied are smoothly varying mean sinusoidal flows and asymmetric mean flows with a large gradient. These types of flow around curved pipes were first studied by Smith (1975). For sinusoidal flow with mean pressure gradient in an arbitrary cross-section large-curvature pipe $\left(R_{c} / D_{c} \gg 1\right.$, where $D_{c}$ is the non-circular cross-sectional dimension), Smith (1975) showed the secondary flow patterns are similar to Lyne's quadrupole vortices under the oscillatory limit $\left(D / \delta_{s} \gg 1\right)$ and Dean's dipole vortices under the quasi-steady limit $\left(D / \delta_{s} \ll 1\right)$. Sumida et al. (1989) performed a parametric study of the influence of $R e_{b}$ and $D / \delta_{s}$ (in a fixed curved pipe $R_{c} / D=3.8$ ) on the secondary flow during the acceleration and deceleration phase of flow cycle. At low driving frequencies $\left(D / \delta_{s} \gtrsim 1\right)$, the secondary flow in the acceleration phase is similar to that observed for steady condition, but the flow is quite different in the deceleration phase. At a high driving frequency of $D / \delta_{s} \gg 1$, the secondary flow pattern does not change throughout the cycle and is similar to the steady condition where the viscous region is concentrated towards the sidewall. This type of flows was further studied by Sumida (2007) and Jarrahi et al. (2011).

The second important feature of unsteady pipe flow is the force that acts on the bend. Pipe forces can be generally interpreted from the change in the momentum flux $\rho(\boldsymbol{u} \cdot \hat{\boldsymbol{n}}) \boldsymbol{u} A$, along a pipe, where $\rho$ is density, $\boldsymbol{u}$ is velocity, $A$ is pipe area and $\hat{\boldsymbol{n}}$ is the unit normal vector. The curvature's influence leads to a net change in $\boldsymbol{u}$ or momentum that leads to a steady bend force. Other effects are the velocity magnitude change $(\boldsymbol{u} \cdot \hat{\boldsymbol{n}})$ or fluid density change $(\rho A)$ that causes unsteadiness in the pipe flow in the form of localised repeated impulsive bend loading. This type of impulsive loading generates sudden shock in the offshore structures (due to ocean-wave slamming (Ghadirian \& Bredmose 2019)) and oscillation in the piping system (due to slug flow (Xiao et al. 2019)) and is the source of a rigid body and vibratory motion in all industrial structures. A major source of impulsive unsteadiness in the energy sector is either a change in the fluid density in multiphase flow (Hou et al. 2014; Tay \& Thorpe 2014; Xiao et al. 2019) or a change in the volume flux from the reciprocating pump (API 618, API 688). Tay \& Thorpe (2014) and Xiao et al. (2019) developed a one-dimensional model based on an integral momentum approach to evaluate the unsteady forces on a pipe bend due to gas slugs along pipes and around pipe bends, where the change in momentum flux arose from the change in fluid volume $(V)$ during slug movement.

While research into flow in curved pipes is quite mature (see figure 1), there are two outstanding gaps in this area. The first concerns the pipe bend force, particularly the 
contribution from flow acceleration. The second gap concerns the separate and distinct contributions from steady and oscillatory components to the secondary flow, which is especially important when the rms velocity is comparable to the mean velocity. A tandem approach of analytical and numerical techniques is applied to analyse these two issues. For the bend force case, a momentum integral approach is applied, which can be used to derive closed-form expressions for high-St and be tested numerically. The non-linearity of inertially-dominated flows complicates the secondary flow analysis, so we develop a linear model by extending Hawthorne's (1951) inviscid analysis to include viscous effects and no-slip condition. For the first time, this technique permits an understanding of the origin of vorticity associated with the secondary flow, which has contributions from the no-slip condition (which creates a source on the wall) and an internal source (which comes from an inviscid stretching process). This analysis is compared to numerical simulations at finite pipe curvature.

The paper is structured as follows: in $§ 2$, the mathematical model is defined, discussing the problem abstraction, methodology, and boundary conditions. To understand the physics, a series of analytical models are discussed in $\S 3$ for the boundary layer oscillation, secondary flow evolution, and pipe bend forces. Drawn by the large parameter space to analyse, we describe in detailed the case of $R e_{b}=2000, S t=1$, and $R_{c} / D=1.5$ regarding the kinematic (§4) and dynamic aspects (§5) of the flow.

\section{Mathematical Model}

\subsection{Fluid Mechanical Model}

The three-dimensional fluid flow $\boldsymbol{u}$ is driven by the gradient of a pressure field $p$ and resisted by viscous stresses $\boldsymbol{\tau}\left(=\mu\left(\nabla \boldsymbol{u}+(\boldsymbol{\nabla} \boldsymbol{u})^{t}\right)\right)$. The dynamics of an incompressible Newtonian fluid are described by the differential forms of the conservation of momentum and mass:

$$
\rho\left(\frac{\partial \boldsymbol{u}}{\partial t}+(\boldsymbol{u} \cdot \nabla) \boldsymbol{u}\right)=-\nabla p+\nabla \cdot \boldsymbol{\tau}, \quad \boldsymbol{\nabla} \cdot \boldsymbol{u}=0,
$$

where $\rho$ is the fluid density. The secondary flow depends on the presence of internal vorticity that comes from the form of the inlet flow and wall conditions. The velocity field is set on the pipe inlet through uniform or fully-developed pipe flow analysis (B 9) based on actual pulsatory volume flux,

$$
Q(t)=\int u \mathrm{~d} A=A U_{b}\left|\sin \Omega_{p} t\right|
$$

and by imposing a kinematic constraint on the velocity at the pipe wall surface, where $U_{b}$ is maximum bulk velocity, $\Omega_{p}$ is angular frequency, and $A$ is pipe cross-sectional area (see figure $2 a$ ). On the pipe outlet surface, the pressure is set at $p=0$.

\subsection{Key parameters}

The pipe flow is characterized by the instantaneous Reynolds number $(R e)$ and maximum Reynolds number $\left(R e_{b}\right)$, defined by

$$
R e=\frac{\left|u_{m}\right| D}{\nu}, \quad R e_{b}=\frac{t_{\nu}}{t_{a}}=\frac{U_{b} D}{\nu}
$$

where $t_{\nu}\left(=D^{2} / \nu\right)$ and $t_{a}\left(=D / U_{b}\right)$ are viscous diffusion and advection time scales, respectively, and $\nu$ is kinematic viscosity. The pulsatory flow (2.2) generates a velocity 


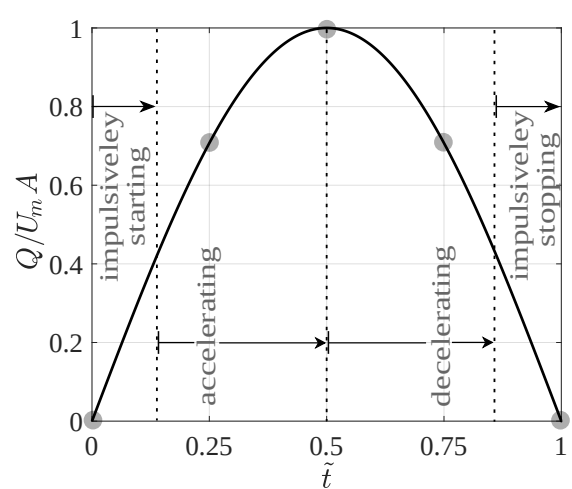

(a)

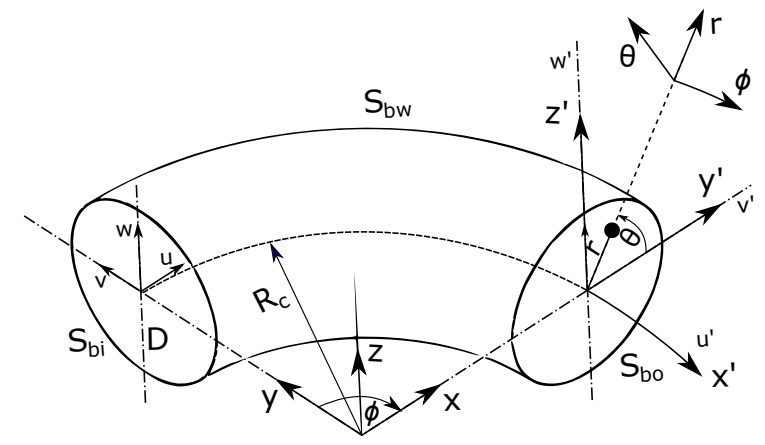

(c)

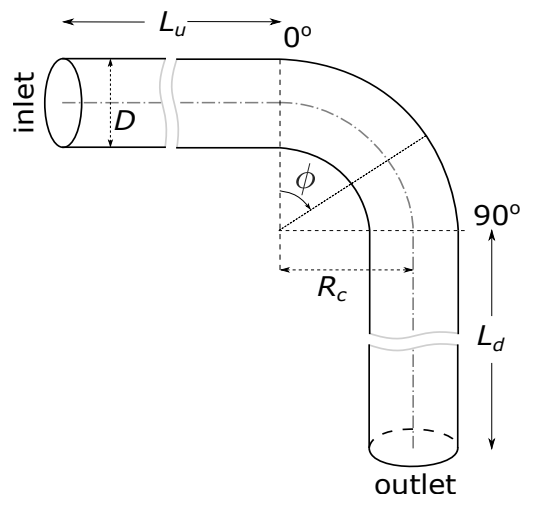

(b)

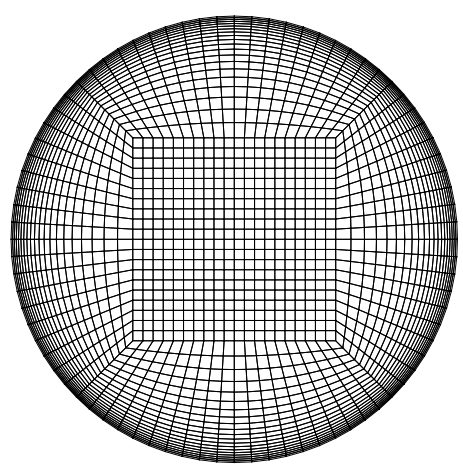

(d)

Figure 2. (a) The variation of the inlet volume flux $\left(Q / U_{b} A\right)$ with dimensionless time $\left(\widetilde{t}=\Omega_{p} t / \pi\right)$ is shown for one cycle. The four phases of one pumping cycle are impulsivelystarting $(R e \ll 1, \dot{Q}>0)$, accelerating $(\operatorname{Re} \gg 1, \dot{Q}>0)$, decelerating $(\operatorname{Re} \gg 1, \dot{Q}<0)$, and impulsively-stopping $(R e \ll 1, \dot{Q}<0)$. (b) A schematic of the computational domain is shown along with the geometrical length scales. $(c)$ The radial $(r)$, azimuthal $(\theta)$, and streamwise $(\phi)$ coordinates are shown. The total pipe bend surface is $S_{b}\left(=S_{b w}+S_{b i}+\right.$ $\left.S_{b o}\right)$ and includes the curved wall $\left(S_{b w}\right)$, inlet $\left(S_{b i}\right)$ and outlet $\left(S_{b i}\right)$ surfaces. $(d)$ The structured non-uniform mesh, shown for a slice normal to the pipe axis, is extruded along the pipe length.

profile (see figure 13), which has the contribution from both anoscillatory boundary layer, of approximate thickness

$$
\delta_{s}=\sqrt{\frac{2 \nu \pi}{\Omega_{p}}},
$$

and steady core flow (characteristic thickness $\delta \sim D$ ). The relative size of the oscillatory boundary layer compared to the pipe diameter $\left(D / \delta_{s}\right)$ gives an appropriate measure of grid resolution next to the wall. The Strouhal number expresses the ratio of advective time scale $t_{a}$ to oscillatory time scale $t_{p}\left(=2 \pi / \Omega_{p}\right)$

$$
S t=\frac{t_{a}}{t_{p}}
$$


and is linked to $\operatorname{Re}_{b}$ and $D / \delta_{s}$ through

$$
\frac{D}{\delta_{s}}=\left(\frac{t_{\nu}}{t_{p}}\right)^{1 / 2}=S t^{1 / 2} R e_{b}^{1 / 2}
$$

The steady boundary layer ratio to oscillatory boundary layer thickness scales approximately as $\delta / \delta_{s} \sim S t^{1 / 2} R e_{b}^{1 / 2}$ so that as $S t$ increases, the oscillatory boundary becomes thinner. The regime plot in the figure 1 shows iso-contours of $S t$ with most numerical studies being in the range $10^{-2}<S t<10^{-1}$. The analysis in this study is focused on high-St inertially-dominated flows with most attention paid to $R e_{b}=2000$ and $S t=1$.

The pipe geometry (figure $2 b$ ) consists of a 90-degree bend connected with straight pipe sections. The curvature ratio $R_{c} / D$ was varied over the range 1 to 10 . The pipe bend is connected to the upstream and downstream pipes, whose lengths are $L_{u} / D(=3)$ and $L_{d} / D(=3)$, respectively. The total pipe length is $L_{t}=L_{u}+\frac{\pi}{2} R_{c}+L_{d}$.

\subsection{Numerical Implementation}

Equations (2.1 a,b) were solved on a well-resolved grid configuration using a wellvalidated finite-volume method within ANSYS FLUENT v19.0 (see supplementary document for verification and validation). A time-step $\Delta t$ was chosen from the constraint $\left(U_{b} \Delta t / h=0.5\right)$ to satisfy the Courant-Friedrichs-Lewy criterion for global stability.

\section{Analytical Model}

\subsection{Boundary layer analysis in an upstream straight pipe section}

The first stepping stone is to analyse the unsteady boundary layer structure in the absence of a bend. We start with the assumption of incompressible one-dimensional Navier-Stokes equation in cyclindrical-polar coordinate system $(r, \theta, x)$ along the axial direction $(x)$ (see figure $2 b, c$ ) in an infinitely long upstream pipe (uniform flow or axially invariant or fully developed pipe flow), with zero swirl $\left(u_{\theta}=0, u_{r}=0, u=u(r, t)\right.$, cross-section spatially invariant pressure $p\left(\frac{\partial p}{\partial r}=0\right.$, hence axial pressure gradient is $\mathrm{d} p(x, t) / \mathrm{d} x$ ), and boundary conditions (Das \& Arakeri 2000) as,

$$
\begin{gathered}
\frac{\partial u}{\partial t}=-\frac{1}{\rho} \frac{\partial p}{\partial x}+\nu\left(\frac{\partial^{2} u}{\partial r^{2}}+\frac{1}{r} \frac{\partial u}{\partial r}\right), \\
u(D / 2, t)=0, \quad \text { and } \frac{\partial u(0, t)}{\partial r}=0 .
\end{gathered}
$$

When the pipe inlet volume flow rate is known,

$$
\int_{0}^{D / 2} 2 \pi r u(r, t) \mathrm{d} r=\frac{\pi}{4} D^{2} u_{m}(t)=Q(t),
$$

where $r=\left.r\right|_{\theta=0}=y-R_{c}$ for upstream straight pipe, $u_{m}(t)$ is cross-sectional averaged velocity (or bulk velocity or flow rate). The streamwise boundary layer flow $u_{\phi}$ can be written as the sum of steady and oscillatory components as:

$$
u_{\phi}=u_{\phi, s}+u_{\phi, o}
$$

Similarly, the azimuthal vorticity comprises of steady and oscillatory components:

$$
\omega_{\theta}\left(=-\frac{\partial u_{\phi}}{\partial r}\right)=\omega_{\theta, s}+\omega_{\theta, u}=-\frac{\partial u_{\phi, s}}{\partial r}-\frac{\partial u_{\phi, o}}{\partial r} .
$$


For a pulsatory flow (2.2), the analytical boundary layer solution based on a given inlet volumetric flux is derived in Appendix B (eq. B 9, where $u_{\phi, s}=u_{1}(r, t), u_{\phi, o}=u_{2}(r, t)$ ). For $t \rightarrow \infty$, the non-dimensional form of $u_{\phi}$ and $\omega_{\theta}(3.3$ and 3.4) are

$$
\begin{gathered}
\widetilde{u}_{\phi}=2 \frac{\bar{u}_{m}}{U_{b}}\left(1-4 \widetilde{r^{*}}\right) \\
-\frac{1}{2} \frac{U_{m}}{U_{b}} \sum_{n=1}^{\infty} a_{n}\left(\frac{J_{0}\left(\gamma_{a}\right)-J_{0}\left(2 \widetilde{r^{*}} \gamma_{a}\right)}{J_{2}\left(\gamma_{a}\right)} e^{i n 2 \pi S t \tilde{t}}+\frac{J_{0}\left(\gamma_{b}\right)-J_{0}\left(2 \widetilde{r^{*}} \gamma_{b}\right)}{J_{2}\left(\gamma_{b}\right)} e^{-i n 2 \pi S t \tilde{t})}\right. \\
\widetilde{\omega}_{\theta}\left(=-\frac{D}{U_{b}} \frac{\partial u_{\phi}}{\partial r}\right)=16 \frac{\bar{u}_{m}}{U_{b}} \widetilde{r^{*}} \\
-\frac{U_{m}}{U_{b}} \sum_{n=1}^{\infty} a_{n}\left(\frac{\gamma_{a} J_{1}\left(2 \widetilde{r^{*}} \gamma_{a}\right)}{J_{2}\left(\gamma_{a}\right)} e^{i n 2 \pi S t \tilde{t}}+\frac{\gamma_{b} J_{1}\left(2 \widetilde{r^{*}} \gamma_{b}\right)}{J_{2}\left(\gamma_{b}\right)} e^{-i n 2 \pi S t \tilde{t}}\right)
\end{gathered}
$$

where

$$
\widetilde{r^{*}}=r / D, \quad \gamma_{a}=(n \pi S t R e)^{1 / 2} \frac{(-1+i)}{2}, \quad \gamma_{b}=(n \pi S t R e)^{1 / 2} \frac{(1+i)}{2} .
$$

\subsection{Secondary flow in the pipe bend}

Secondary flows can be analysed from a $p-\boldsymbol{u}$ (Singh 1974; Yao \& Berger 1975; Smith 1976 ) or a $\boldsymbol{\omega}$ perspective (Hawthorne 1951; Lyne 1971; Agrawal et al. 1978; Olson \& Snyder 1985). Using these approaches, several new studies have categorised the secondary flow pattern under asymmetric pulsatory pipe flows (Boiron et al. 2007; Krishna et al. 2017; Najjari et al. 2019). For a starting-waiting flow (single positive pulse followed by a waiting period), both Boiron et al. (2007) and Krishna et al. (2017) have interpreted the generation of a secondary flow using $p-\boldsymbol{u}$ approach due to the imbalance in the centrifugal force and the radial pressure gradient causing the flow to be driven from the inner to the outer bend and azimuthal return flow from outer to inner bend. The $p-\boldsymbol{u}$ approach has an appealing physical interpretation but is complicated to solve analytically.

On the other hand, a $\boldsymbol{\omega}$ approach is generally more complicated to interpret but easier to solve analytically. Most recent studies have used vorticity or integral measures, such as circulation, as diagnostic tools to distinguish between different types of secondary motion (Najjari et al. 2019). Kartik \& Michael (2018) used circulation to understand the morphological changes in the large-scale coherent structures. Najjari et al. (2019) looked at the various terms in the vorticity equation to analyse Lyne-type vortex production and the influence of slip and no-slip wall conditions.

The generation of a secondary flow is associated with the streamwise component of vorticity $\left(\omega_{\phi}\right)$ whose evolution with time is described by:

$$
\frac{\partial \omega_{\phi}}{\partial t}+(\boldsymbol{u} \cdot \nabla) \omega_{\phi}=(\boldsymbol{\omega} \cdot \nabla) u_{\phi}+\nu \nabla^{2} \omega_{\phi}
$$

The first term on the right-hand-side of (3.8) describes an inviscid stretching process, discussed by Hawthorne (1951), Lighthill (1956) and Hunt (1973), caused by the spatial gradients of $u_{\phi}$. This provides the source for the production of $\omega_{\phi}$ :

$$
S=(\boldsymbol{\omega} \cdot \nabla) u_{\phi}=\frac{\omega_{\theta}}{r} \frac{\partial u_{\phi}}{\partial \theta}+\omega_{r} \frac{\partial u_{\phi}}{\partial r}+\frac{\omega_{\phi}}{R_{c}} \frac{\partial u_{\phi}}{\partial \phi},
$$

through the tilting of the azimuthal and radial vorticity $\left(\omega_{\theta}, \omega_{r}\right)$ by cross-stream varia- 
tions of $u_{\phi}$ (first two terms of 3.9) and the compression of $\omega_{\phi}$ by a streamwise variation of $u_{\phi}$ (last term of 3.9 ).

The analysis of Dean (1928) and Lyne (1971) are based on small-curvature pipes where the cross-stream strain is approximated by

$$
\nabla u_{\phi}=\frac{\gamma}{R_{c}} \frac{\partial u_{\phi}}{\partial y^{\prime}} \hat{\boldsymbol{c}}^{\prime}
$$

$(\gamma=2)$, and this leads to a simplification of the source term

$$
S=(\boldsymbol{\omega} \cdot \nabla) u_{\phi}=\frac{\gamma}{R_{c}}\left(\omega_{\theta} u_{\phi} \sin \theta+\omega_{r} u_{\phi} \cos \theta\right) .
$$

Dean (1928)[eq. 11] and Lyne (1971)[eq. 2.14] studied the secondary flow generated in an infinitely long pipe, where both sources in (3.11) are important. For an infinitely long pipe, the self-induced motion of the secondary flow is taken into account in the advection term, but streamwise advection is neglected (since $\partial / \partial \phi=0$ ). However, near the bend inlet (where $\left|\omega_{r}\right| \ll\left|\omega_{\theta}\right|$ ), the dominant source term arises from stretching of $\omega_{\theta}$,

$$
S \simeq \frac{\gamma}{R_{c}} \omega_{\theta} u_{\phi} \sin \theta
$$

and $\omega_{\phi}$ is primarily advected by the streamwise flow. This leads to a simplification and linearisation of $(3.8)$ :

$$
\frac{\partial \omega_{\phi}}{\partial t}+u_{\phi} \frac{\partial \omega_{\phi}}{\partial s}=\frac{\gamma \omega_{\theta} u_{\phi}}{R_{c}} \sin \theta+\nu \nabla^{2} \omega_{\phi}
$$

The simplicity of this linear model is that it allows the contributions from the inlet azimuthal vorticity, steady core and oscillatory boundary layers to $\omega_{\phi}$ to be understood and quantified. As $\phi$ increases, the self-induced motion created by the secondary flow becomes more important, and its influence needs to be taken in to account.

The steady inviscid result of Hawthorne (1951, eq. 12) and Olson \& Snyder (1985, eq. 3 ) can be recovered from (3.13) by noting that the streamwise vorticity after a distance $s$, along the pipe, is

$$
\omega_{\phi} \simeq \int_{0}^{s} \frac{S}{u_{\phi}} \mathrm{d} s \simeq \gamma \phi \omega_{\theta i} \sin \theta
$$

where $\omega_{\theta i}$ is the inlet azimuthal vorticity field. For a pulsatory flow, the source term averaged over a period of $2 \pi / \Omega$ is

$$
\bar{S}=\frac{\gamma}{R_{c}}\left(P_{s s}+P_{o o}\right) \sin \theta,
$$

(where the overbar represents a time average) has separate contributions from the steady core and oscillatory components of the flow:

$$
P_{s s}=\omega_{\theta, s} u_{\phi, s}, \quad P_{o o}=\overline{\omega_{\theta, o} u_{\phi, o}} .
$$

The numerical solution to (3.13) is discussed in Appendix C.

The linear model provides a framework to understand how the secondary flow grows in the near bend inlet region and the distinct contributions from steady and oscillatory flow components to $\omega_{\phi}$. The framework extends Hawthorne's (1951) inviscid analysis to include viscous diffusion and distinguishes between the distinct contributions from inviscid stretching and the no-slip condition to $\omega_{\phi}$. Since the model neglects non-linear processes, such as self-induced motion and stretching of $\omega_{r}$, its validity is limited to the near bend inlet region and small-curvature pipes. 


\subsection{Forces}

The force on the pipe bend (A 4) can be calculated directly from the integral of pressure and viscous stresses over $S_{b w}$. It is more insightful to re-expressing the force in terms of an integral over the pipe bend inlet and outlet surfaces $\left(S_{b i}\right.$ and $\left.S_{b o}\right)$ (see figure $2 c$ ), and the pipe bend volume $V$ using the momentum integral approach (Yunus 2017; Potter et al. 2016; White 2016), to give

$$
\boldsymbol{F}_{p}=-\int_{S_{b i}+S_{b o}} p \hat{\boldsymbol{n}} \mathrm{d} S-\rho\left(\int_{V} \frac{\partial \boldsymbol{u}}{\partial t} \mathrm{~d} V+\int_{V}(\boldsymbol{u} \cdot \nabla) \boldsymbol{u} \mathrm{d} V\right)+\int_{S_{b i}+S_{b o}} \boldsymbol{\tau} \cdot \hat{\boldsymbol{n}} \mathrm{d} S .
$$

When the outlet surface $\left(S_{b o}\right)$ is at an angle $\phi$ with respect to the inlet surface $\left(S_{b i}\right)$ the force integral can be written as

$$
\begin{array}{r}
\boldsymbol{F}_{p}\left(=\left(F_{x}, F_{y}\right)\right)=-\left(p_{b i} A+\rho \int_{S_{b i}} u_{\phi}^{2} \mathrm{~d} A+\rho \int_{S_{b o}} u_{\phi} v^{\prime} \mathrm{d} A\right) \hat{\boldsymbol{n}}_{i} \\
-\left(p_{b o} A+\rho \int_{S_{b o}} u_{\phi}^{2} \mathrm{~d} A+\rho \int_{S_{b i}} u_{\phi} v^{\prime} \mathrm{d} A\right) \hat{\boldsymbol{n}}_{o}-\rho \int_{V} \frac{\partial \boldsymbol{u}}{\partial t} \mathrm{~d} V-\int_{S_{b i}+S_{b o}} \boldsymbol{\tau} \cdot \hat{\boldsymbol{n}} \mathrm{d} S
\end{array}
$$

where $p_{b i}$ and $p_{b o}$ are bend inlet and outlet pressure and $\hat{\boldsymbol{n}}_{i}(=(-1,0)), \hat{\boldsymbol{n}}_{o}(=$ $(\cos \phi,-\sin \phi))$ are unit vectors normal to the pipe bend inlet and outlet. $F_{x}$ is quite different from the $F_{y}$ expression. We anticipate, and indeed find, that $F_{x}$ and $F_{y}$ are different. Tay \& Thorpe (2014) and Xiao et al. (2019) applied a momentum approach to evaluate the unsteady forces on a pipe bend due to slugs passing, where the fluid volume $(V)$ changed with time due to the slug movement, but the volume flux $(Q)$ was constant. In our example, the fluid volume $(V)$ is constant, but volume flux $(Q)$ changed with time.

The velocity field in a curved pipe can be written as the summation of three components - streamwise $(\hat{\boldsymbol{s}})$, crosswise $(\hat{\boldsymbol{c}})$ and spanwise $(\hat{\boldsymbol{a}})$, as

$$
\boldsymbol{u}=u_{\phi} \hat{\boldsymbol{s}}+v^{\prime} \hat{\boldsymbol{c}}+w^{\prime} \hat{\boldsymbol{a}}, \quad \frac{\mathrm{d} \boldsymbol{u}}{\mathrm{d} t}=\frac{\mathrm{d} u_{\phi}}{\mathrm{d} t} \hat{\boldsymbol{s}}+\frac{\mathrm{d} v^{\prime}}{\mathrm{d} t} \hat{\boldsymbol{c}}+\frac{\mathrm{d} w^{\prime}}{\mathrm{d} t} \hat{\boldsymbol{a}},
$$

where the tangential unit vector is $\hat{\boldsymbol{s}}(=(\cos \phi,-\sin \phi))$. The velocity components scale as $u_{\phi} \sim u_{m}$ and $v^{\prime}, w^{\prime} \sim \lambda u_{m}$, where $\lambda \sim U_{b}^{2} D^{2} \kappa^{2} /\left(2 R_{c}^{3} \Omega_{p}\right)$ (eq. 4.13 in Krishna et al. (2017)). The typical values of $\lambda$ are $\lambda<1$ and $\lambda \ll 1$ for $D / \delta_{s}>0$ and $D / \delta_{s} \gg 0$, respectively. This means that the force due to the secondary flow is small compared to the contribution from the streamwise components. Therefore,

$$
\begin{array}{r}
\boldsymbol{F}_{p}=\underbrace{-\left(\bar{p}_{b i}+\rho \overline{u_{\phi i}^{2}}\right) A \hat{\boldsymbol{n}}_{i}-\left(\bar{p}_{b o}+\rho \overline{u_{\phi o}^{2}}\right) A \hat{\boldsymbol{n}}_{o}-\rho \frac{\mathrm{d} u_{m}}{\mathrm{~d} t} \int_{V} \hat{\boldsymbol{s}} \mathrm{d} V}_{\text {streamwise components }} \\
-\underbrace{\rho A\left(\overline{u_{\phi i} v_{i}^{\prime}} \hat{\boldsymbol{n}}_{i}+\overline{u_{\phi o} v_{o}^{\prime}} \hat{\boldsymbol{n}}_{o}\right)-\rho \int_{V} \frac{\mathrm{d}\left(v^{\prime} \hat{\boldsymbol{c}}+w^{\prime} \hat{\boldsymbol{a}}\right)}{\mathrm{d} t} \mathrm{~d} V}_{\text {crosswise components }}-\int_{S_{b i}+S_{b o}} \boldsymbol{\tau} \cdot \hat{\boldsymbol{n}} \mathrm{d} S,
\end{array}
$$

where the overbar represents the cross-sectional averaged quantities. The volume flux across the pipe is constant along the pipe length due to incompressibility, and hence both $\int u_{\phi} \mathrm{d} A$ and $\frac{\mathrm{d}}{\mathrm{d} t} \int u_{\phi} \mathrm{d} A$ are independent of $\hat{\boldsymbol{s}}$. So, we can set

$$
\bar{u}_{\phi o}=\bar{u}_{\phi i}=u_{m}(t), \quad \frac{\mathrm{d} \bar{u}_{\phi o}}{\mathrm{~d} t}=\frac{\mathrm{d} \bar{u}_{\phi i}}{\mathrm{~d} t}=\frac{\mathrm{d} u_{m}}{\mathrm{~d} t} .
$$


Also,

$$
\rho \overline{u_{\phi i}^{2}}=\gamma_{i} \rho u_{m}^{2} \quad \rho \overline{u_{\phi o}^{2}}=\gamma_{o} \rho u_{m}^{2},
$$

where $\gamma=\overline{u_{\phi}^{2}} / u_{m}^{2}(t)$ depends on the velocity profile across pipe. The dominant terms in (3.20) are identified in the limit of high- $R e_{b}$. The secondary flow generates crosswise momentum flux whose terms scale as $\rho \lambda u_{m}^{2} \rho A$, and since $\lambda \ll 1$ their contribution to the total bend force is small. The contribution from the secondary flow acceleration is expected to scale as $\rho \lambda A R_{c} u_{m} \Omega$, and again, smaller than the contribution from the streamwise flow acceleration and be negligible. The viscous contribution arises from the integration over the inlet and outlet planes and therefore scales as $\mu u_{m} D \sim \rho u_{m}^{2} A / R e_{b}$. As $R e_{b}$ is large, the viscous contribution term is negligible.

At high- $R_{b}$, the main contributions to the bend force are from the deflection of the pipe flow arising from the change in $\hat{\boldsymbol{n}}$ and the integral along the bend (from $\int \hat{\boldsymbol{s}} \mathrm{d} V$ ), with weaker contributions from the secondary flow and viscous effects. The pipe bend force is, to leading order,

$$
\boldsymbol{F}_{p} \simeq-\left(\bar{p}_{b i}+\rho \gamma_{i} u_{m}^{2}\right) A \hat{\boldsymbol{n}}_{i}-\left(\bar{p}_{b o}+\gamma_{o} \rho u_{m}^{2}\right) A \hat{\boldsymbol{n}}_{o}-\rho \frac{\mathrm{d} u_{m}}{\mathrm{~d} t} \int_{V} \hat{\boldsymbol{s}} \mathrm{d} V .
$$

For a pipe with a bend angle of $\phi_{m}$, the pipe bend forces in $x$ and $y$ direction reduced to

$$
\begin{aligned}
& F_{x}=\left(\bar{p}_{b i}-\bar{p}_{b o} \cos \phi_{m}+\gamma_{i} \rho u_{m}^{2}\left(1-\cos \phi_{m}\right)-\rho \frac{\mathrm{d} u_{m}}{\mathrm{~d} t} R_{c} \sin \phi_{m}\right) A, \\
& F_{y}=\left(\left(\bar{p}_{b o}+\gamma_{o} \rho u_{m}^{2}\right) \sin \phi_{m}-\left(\cos \phi_{m}-1\right) \rho \frac{\mathrm{d} u_{m}}{\mathrm{~d} t} R_{c}\right) A .
\end{aligned}
$$

The difference between the bend force in the $x$ - and $y$ directions is

$$
F_{x}-F_{y}=\left(\bar{p}_{b i}-\bar{p}_{b o}+\left(\gamma_{i}-\gamma_{o}\right) \rho u_{m}^{2}-2 \rho \frac{\mathrm{d} u_{m}}{\mathrm{~d} t} R_{c}\right) A,
$$

and arises mainly from the pressure drop along the pipe and the acceleration force (since typically $\gamma_{0} \simeq \gamma_{i}$ ).

In the limit of high- $R e_{b}$ and high-St, the bend force can be estimated by noting that the pressure variation along the whole pipe is dominated by the flow acceleration and the pressure variation across the pipe is small $\left(\bar{p}_{b i} \simeq p_{b i}, \bar{p}_{b o} \simeq p_{b o}\right)$. When the exit pipe pressure is $p_{e}$, this shows that the pressure at a point in the pipe is proportional to the distance to the tube outlet $s_{o}$, i.e.

$$
p=p_{e}+\int_{L}^{s} \nabla p \cdot \mathrm{d} \hat{\boldsymbol{s}}=p_{e}-\rho \frac{\mathrm{d} u_{m}}{\mathrm{~d} t} \hat{\boldsymbol{s}} .
$$

The distance from the pipe bend exit to the pipe exit is $s_{o}=L_{d}$, so that the distance from the pipe bend inlet to the pipe exit is $s_{i}=\phi_{m} R_{c}+L_{d}$. For a pulsatory flow (2.2) driven around a 90 -degree bend $\left(\phi_{m}=\pi / 2\right)$, the $x$-component of force over one cycle of the flow is

$F_{x}=\rho A U_{b}^{2}\left(-S t \frac{2 \pi\left(L_{d}+R_{c}(\pi / 2+1)\right)}{D} \cos \Omega_{p} t+\gamma_{i} \sin ^{2} \Omega_{p} t\right)+p_{e} A, \quad$ for $\quad 0 \leqslant \Omega_{p} t \leqslant \pi$.

From (3.27), the influence of flow acceleration is due to the first term, which dominates over the force due to the momentum flux when

$$
S t>\underbrace{\frac{D}{2 \pi\left(L_{d}+\pi R_{c} / 2+R_{c}\right)}}_{S t_{c r}} .
$$


As the length of the pipe after the bend increases, the pressure in the bend region becomes increases in magnitude and makes a greater contribution to the total bend force. The impact of the pipe length $L_{d}$ on the bend force arises simply due to pressure increasing linearly along the pipe and being proportional to the flow acceleration. The simple analysis shows that the force depends on the length of the outlet pipe for high-St flows. The difference between the bend force in the $x$ - and $y$ directions for a $90^{\circ}$ bend is

$$
F_{x}-F_{y}=\left(-\rho R_{c}\left(\frac{\pi}{2}+2\right) \frac{\mathrm{d} u_{m}}{\mathrm{~d} t}+\left(\gamma_{i}-\gamma_{o}\right) \rho u_{m}^{2}\right) A,
$$

and arises mainly from the pressure drop along the pipe and the acceleration force (since typically $\left.\gamma_{0}=\gamma_{i}\right)$.

\section{Kinematics of the flow field}

The structure of the velocity and vorticity fields are discussed in detail for the case of $R e_{b}=2000, S t=1, R_{c} / D=1.5$ and compared against simulations with a steady inlet flow (with $R e_{b}=2000$ and $S t=0$ ). An inlet velocity distribution based on the analytical solution (B 9) was used, where both mean (steady) and oscillatory component has a comparable contributions to the secondary flow formation. The numerical simulations are first run for 50 cycles $\left(t=50 \pi / \Omega_{p}\right)$, so the initial transient is diminished (see supplementary document for cycle convergence) and the results are reported for the 51 th cycle. The results are cast in terms of the dimensionless time after the $50^{\text {th }}$ cycle, or $\widetilde{t}=\Omega_{p} t / \pi-50$, where $\widetilde{t}$ varies from 0 and 1 .

\subsection{Velocity field}

The three-dimensional flow streamlines are shown in figure $3(a, b, c, d)$, and a color map has been added to them, indicating flow speed. The accelerating $(\tilde{t}=0.25)$ and decelerating $(\widetilde{t}=0.75)$ phases show a similar streamline pattern as the steady flow simulation with the core flow skewed towards the outer bend region. A helical streamline pattern is seen after the bend, which is associated with streamwise vorticity. This pattern becomes more pronounced for the pulsatory case when $\widetilde{t}=0$ because the mean flow is smaller along the pipe.

There is an extensive literature on three-dimensional flow separation - centred around topological shear stress pattern (Hunt et al. 1978; Tobak \& Peake 1982), an MRS criterion (Inoue 1981), distinguishing between flow reversal and separation (Das \& Arakeri 1998), and spatial/temporal pressure gradients (Das et al. 2016; Krishna et al. 2017). Here, the point of flow separation is discussed through viscous stress lines on the pipe surface, which obeys certain topological rules (see Hunt et al. (1978)). Figure 3(e) shows the shear stress lines (with $|\widetilde{\tau}|$ mapping) on the pipe surface for a steady and pulsatory inlet flow conditions and the location of singular points (saddle and nodes) in the stress field are indicated. The surface of separation is evident for the steady inlet flow simulation by a saddle point in the near bend outer wall region (upstream); it can also be identified from a detailed examination of the local streamlines. Flow separation is observed during the decelerating phase $(\widetilde{t}=0.75)$ and is identified by a saddle point after the bend (in the outer wall region). While no separation is observed at $\tilde{t}=0$, flow reversal occurs adjacent to the pipe wall due to the velocity profile inflection at $\widetilde{t}=0$ (see figure $13 c$ ). During the accelerating phase $(\tilde{t}=0.25)$, the flow is accelerating everywhere, suppressing the potential for separation.

For a steady inlet flow, the consequence of high- $R e_{b}$ and large-curvature is that the flow impacts at the bend outer wall generating a weak return flow (in the form of an 


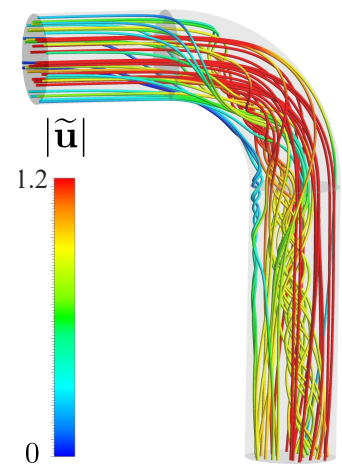

(a)

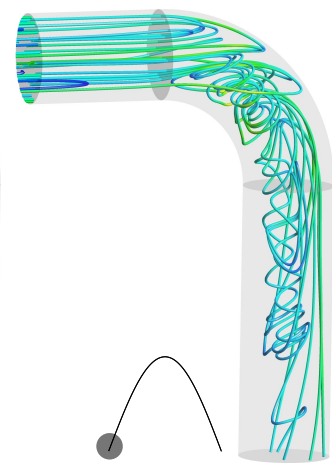

(b)

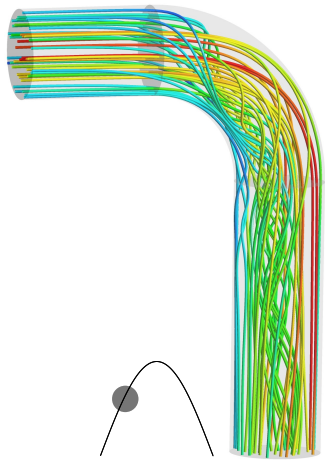

(c)

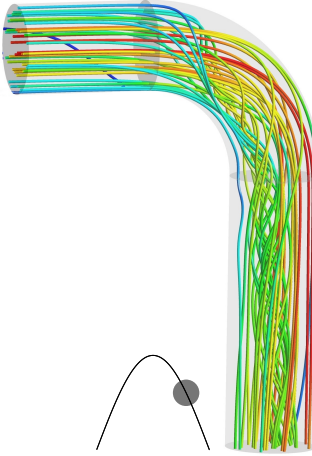

(d)

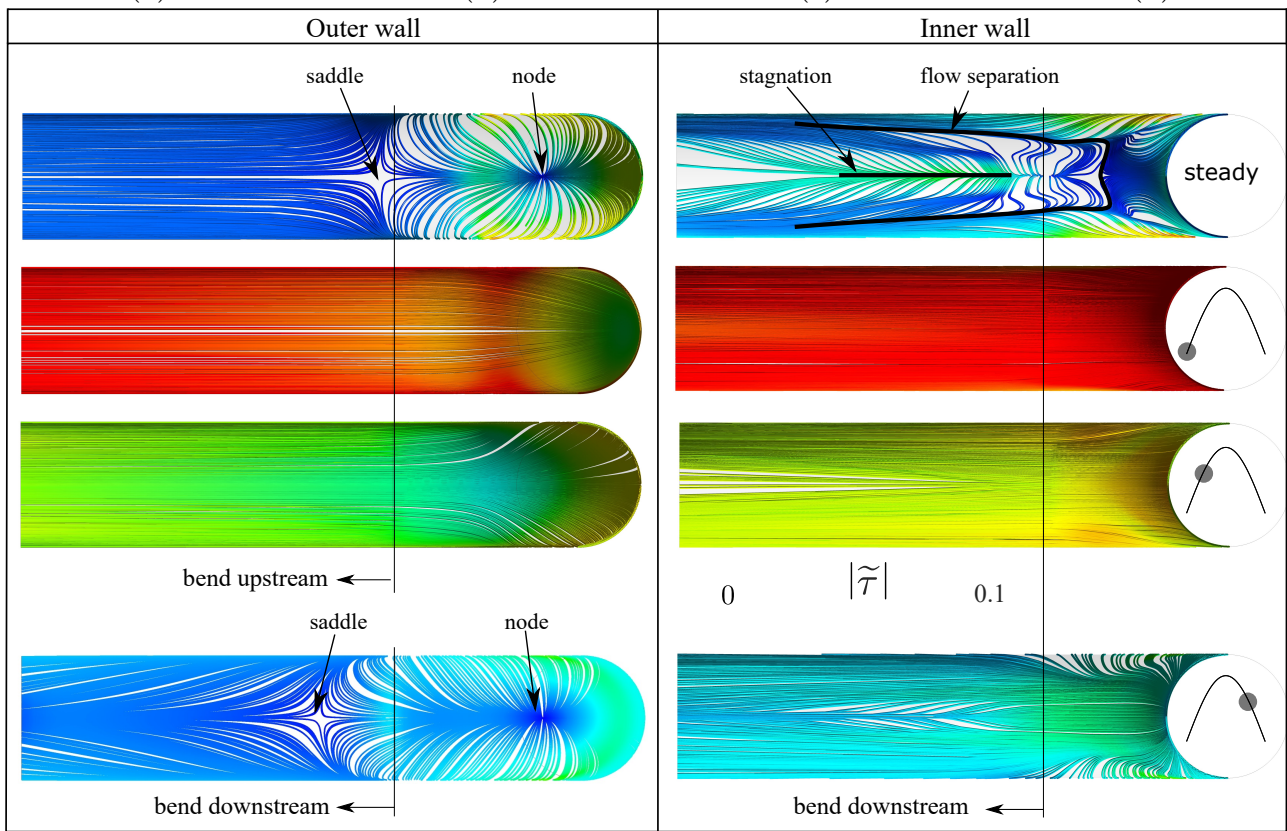

(e)

Figure 3. Comparison of three-dimensional streamlines in $(a)$ a steady inlet flow $\left(R e_{b}=\right.$ 2000 and $S t=0)$ and a pulsatory inlet flow $\left(R e_{b}=2000\right.$ and $\left.S t=1\right)$ in a pipe bend $\left(R_{c} / D=1.5\right)$ corresponding to $(b) \tilde{t}=0,(c) 0.25$ and $(d) 0.75$. (e) Shear stress pattern on the outer wall (left side) and inner wall (right side) for a steady and pulsatory inlet flow conditions.

upstream perturbation), leading to a singular point and as a consequence, flow separation upstream. These are evident as a node on the pipe bend surface in figure 3(e). Smith (1976) identified an anomalous pressure gradient upstream of the pipe bend and on the outer wall in a high- $R e_{b}\left(R e_{b} \gg 1\right)$ small-curvature $\left(R_{c} / D \gg 1\right)$ pipe flow. This perturbation appears to be significant enough at large-curvature to generate separation observed in figure $3(e)$. This effect weakens at lower- $R e_{b}$ and small-curvature, where the saddle point (in the upstream) shifts to the bend region and becomes closer to the node. Weak flow reversal and the upstream perturbation can also be seen in figure $10(c)$ of Krishna et al. (2017). 


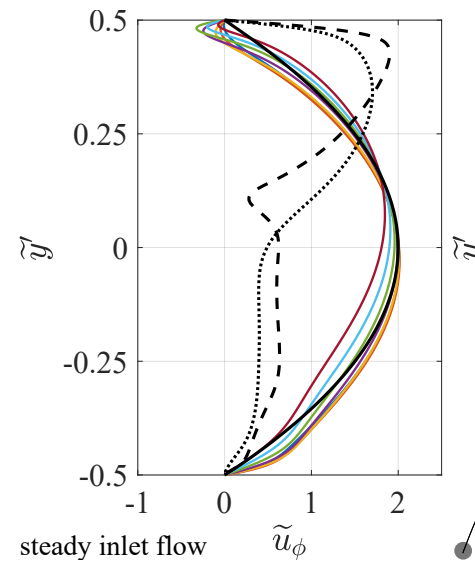

(a)

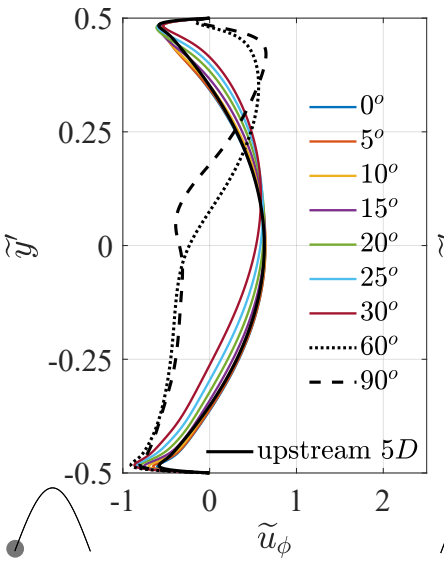

(b)

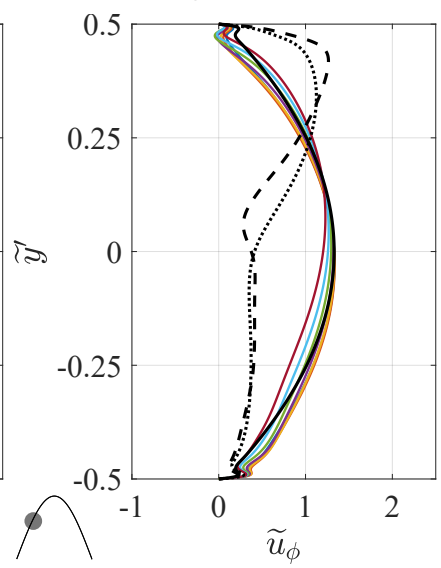

(c)

Figure 4. Profiles showing the instantaneous streamwise velocity in a plane $z=0$ along $y^{\prime}$ at various bend locations. The results are for a $(a)$ steady inlet flow $\left(R e_{b}=2000\right.$ and $S t=0)$ and a pulsatory inlet flow $\left(R e_{b}=2000\right.$ and $\left.S t=1\right)$ in a pipe bend $\left(R_{c} / D=1.5\right)$ corresponding to $(b) \widetilde{t}=0$, and $(c) 0.25$. Upstream data was captured at a distance of $1.5 D$ from the bend inlet.

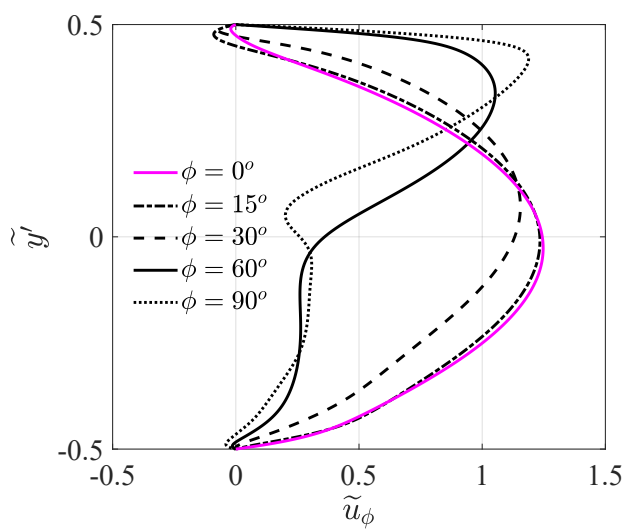

(a)

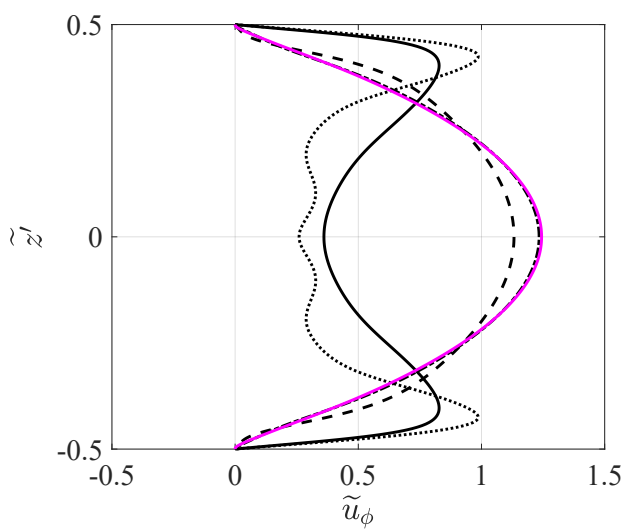

(b)

Figure 5. Variation of time-averaged streamwise velocity $\overline{\widetilde{u}}_{\phi}$ along $(a) \widetilde{y}^{\prime}$ and $(b) \widetilde{z}^{\prime}$ at various bend locations $\left(\phi=0^{0}, 15^{0}, 30^{\circ}, 60^{\circ}\right.$ and $\left.90^{\circ}\right)$. The results are for a pulsatory inlet flow $\left(R e_{b}=2000\right.$ and $\left.S t=1\right)$ in a pipe bend $\left(R_{c} / D=1.5\right)$.

Figure 4 shows a comparison between the velocity profiles for the steady and pulsatory flows. Upstream of the pipe bend, the streamwise velocity profile is symmetric. In the near bend region, the velocity profiles for pulsatory flow case have a similar shape over time but are laterally displaced depending on the instantaneous volume flux. With increasing $\phi\left(\phi>30^{0}\right)$, the flow is skewed towards the outer wall. For a steady inlet flow, the weak negative velocities near the bend inlet (outer wall region) are due to flow separation (see figure $4 a$ that can be identified by the saddle point in figure $3 e$ ). It is worth noting that while the streamwise velocity is positive at the inlet, the effect of the viscous boundary layer leads to flow reversal and a strong gradient of velocity at the wall when $\widetilde{t}=0$ and 


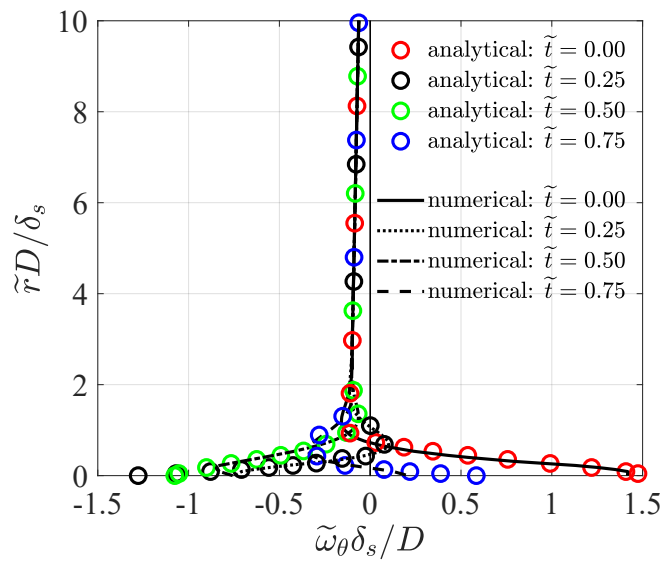

(a)

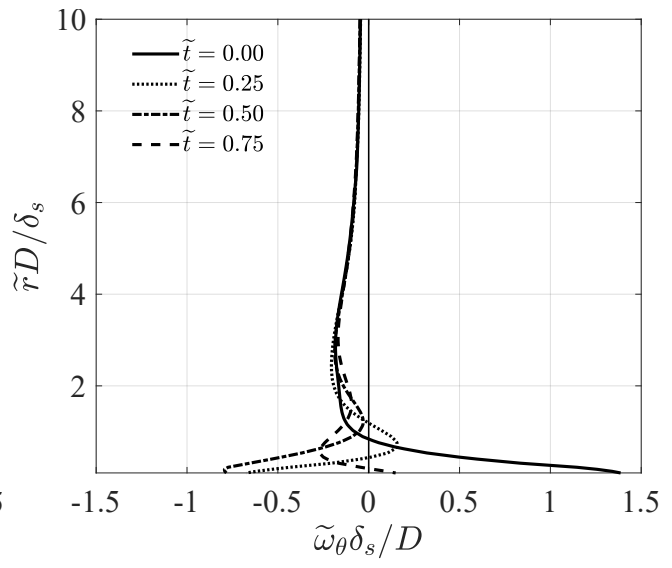

(b)

Figure 6 . The dimensionless azimuthal vorticity $\left(\widetilde{\omega}_{\theta}\right)$ for a pulsatory inlet flow condition ( $R e_{b}=2000, S t=1$, and $\left.R_{c} / D=1.5\right)$ is plotted as a function of distance from the sidewall along the line $\theta=90^{\circ}$ for times $\widetilde{t}=0,0.25,0.50$ and 0.75 . In $(a)$, the results are plotted in the straight upstream pipe - numerically (solid lines) at $1.5 \mathrm{D}$ upstream of bend and analytically (circular markers) based on (3.4). In (b), the numerical results are shown for pipe bend region at $\phi=30^{0}$.

$u_{m}=0$. This is especially prominent in figure $4(b)$, with no evidence of flow separation seen in figure $3(e)$.

The time-averaged streamwise velocity along $\widetilde{y}^{\prime}$ (the line starting extending from the inner to the outer wall) and $\widetilde{z}^{\prime}$ (the line starting extending from the bottom to top sidewall) at bend locations are shown in figure $5(a, b)$, respectively. The variation of $\overline{\widetilde{u}}_{\phi}$ along $\widetilde{z}^{\prime}$ shows that the streamwise velocity is approximately parabolic in the near bend inlet region $\left(\phi<30^{0}\right)$.

\subsection{Vorticity field}

\subsubsection{Azimuthal vorticity}

In a straight pipe section, the azimuthal vorticity for a pulsatory inlet flow condition has two distinct components $(3.4)$ - a steady component $\left(\widetilde{\omega}_{\theta, s}\right)$ in the steady boundary layer (thickness, $\delta$ ) and an oscillatory component $\left(\widetilde{\omega}_{\theta, u}\right)$ in the Stokes boundary layer (thickness, $\left.\delta_{s}\right)$. In figure $6(a)$, the linear model of $\widetilde{\omega}_{\theta}(3.4)$ consists of a slow decay (negative) associated with the steady boundary vorticity (over distance $\delta$ ) and rapid oscillatory decay (over distance $\delta_{s}$ ). These analytical results are captured quite well in the numerical results with high-resolution grid at $1.5 \mathrm{D}$ upstream of pipe inlet (see figure $6 a$ ). The effect of diffraction and distortion of primary $\widetilde{\omega}_{\theta}$ can be seen in the bend region at $\phi=30^{\circ}$, where the profiles of $\widetilde{\omega}_{\theta}$ changes (see figure $6 b$ ).

\subsubsection{Source of streamwise vorticity $\overline{(S})$}

The time-averaged source of $\omega_{\phi}, \bar{S}$, is proportional to the sum of $\widetilde{P}_{s s}$ and $\widetilde{P}_{o o}$, which are proportional to the production of streamwise vorticity from the steady and oscillatory components of the flow, and these are discussed in the figure 7. Figure $7(a)$ shows a comparison of the average of $\widetilde{P}=\tilde{\omega}_{\theta} \widetilde{u}_{\phi}$ over one period for a range of $S t$ spanning steady and pulsatory flows. This shows that the source term (which is proportional to $\widetilde{P}$ ) for steady and high-Strouhal-numbers are similar except within the thin Stokes layer. 


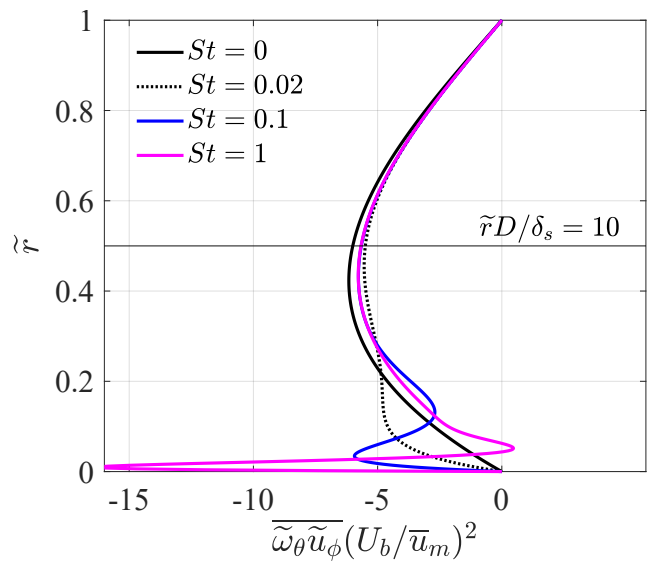

(a)

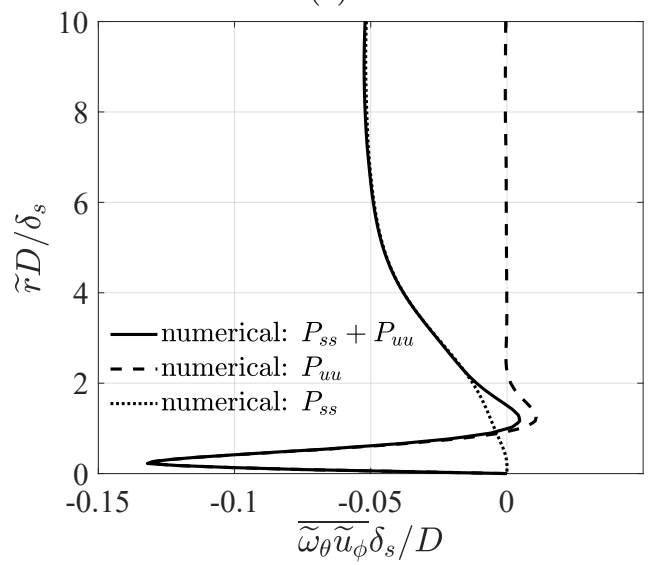

(c)

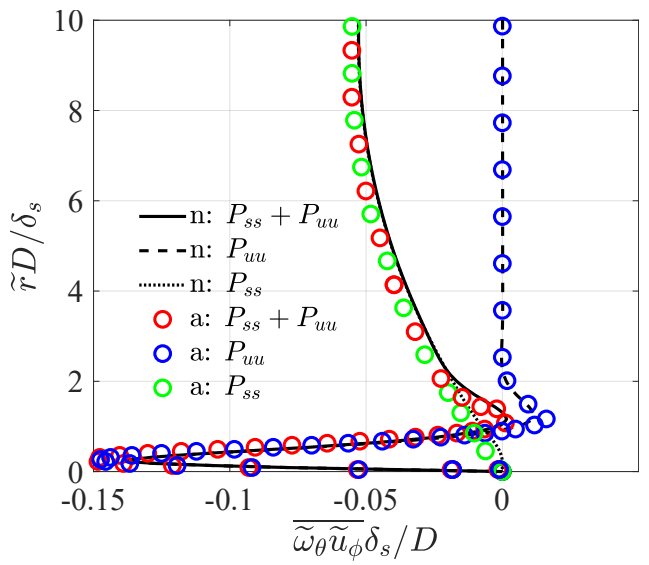

(b)

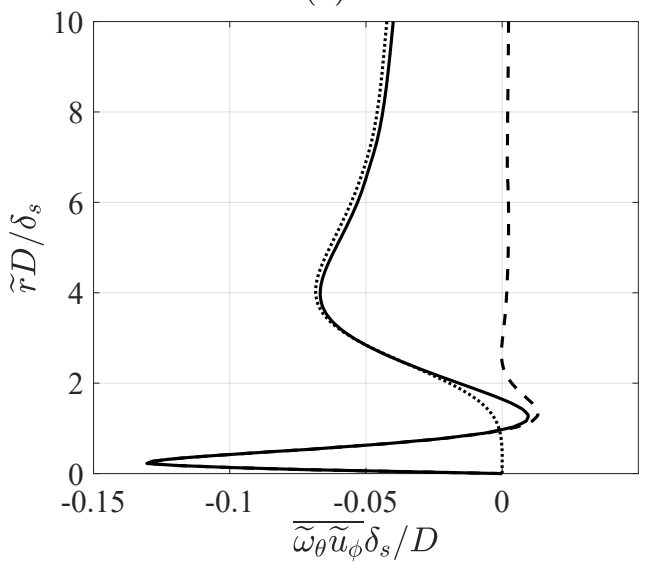

(d)

Figure 7. Spatial distribution of dimensionless time-averaged production terms plotted against the distance from the sidewall along the line $\theta=90^{\circ}$. (a) A comparison of time-

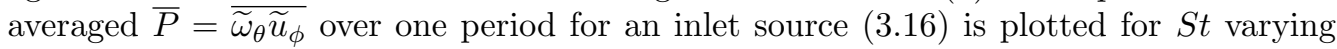
from steady inlet flow $\left(R e_{b}=2000\right.$ and $\left.S t=0\right)$ to high- $S t$ limit $\left(R e_{b}=2000\right.$ and $\left.S t=1\right)$. (b) The numerical results (solid lines) at the upstream of bend (captured at 1.5D) are compared with the analytical results (circular markers). The numerical results are also shown in the bend region at $(c) \phi=15^{0}$ and $(d) \phi=30^{\circ}$. The results in $b, c, d$ are for a pulsatory inlet flow $\left(R e_{b}=2000\right.$ and $\left.S t=1\right)$ in a pipe bend $\left(R_{c} / D=1.5\right)$.

Figure $7(b)$ shows a comparison between $\widetilde{P}$ evaluated from the numerical flow simulations and the analytical predictions. The observations confirm the slow variation of the single signed $\widetilde{P}_{s s}$ over $\delta$, while $\widetilde{P}_{o o}$ is larger in magnitude but decays rapidly over distance $\delta_{s}$ from the wall. The total production term $\widetilde{P}$ has a small negative dip, beyond the maximum. The total production term is numerically evaluated (using 3.16) at $1.5 \mathrm{D}$ upstream of bend (quite consistent with analytical model (see figure $7 b$ )) and at bend locations $\phi=15^{0}$ (see figure $7 c$ ) and $\phi=30^{0}$ (see figure $7 d$ ). The decomposition of $\widetilde{P}$ into $\widetilde{P}_{s s}$ and $\widetilde{P}_{o o}$ exploited the slow change in $\widetilde{P}_{s s}$ and $\widetilde{P}_{o o}=\widetilde{P}-\widetilde{P}_{s s}$. The essential character of the analytical model is confirmed. 
High-Strouhal-number pulsatory flow in a curved pipe

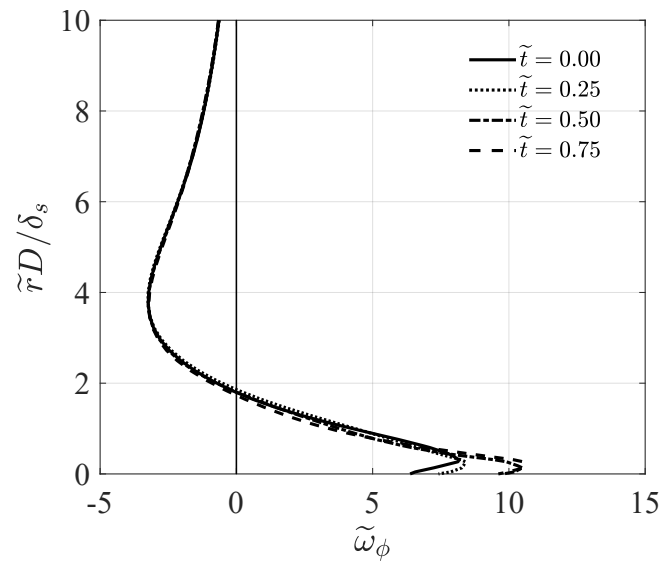

(a)

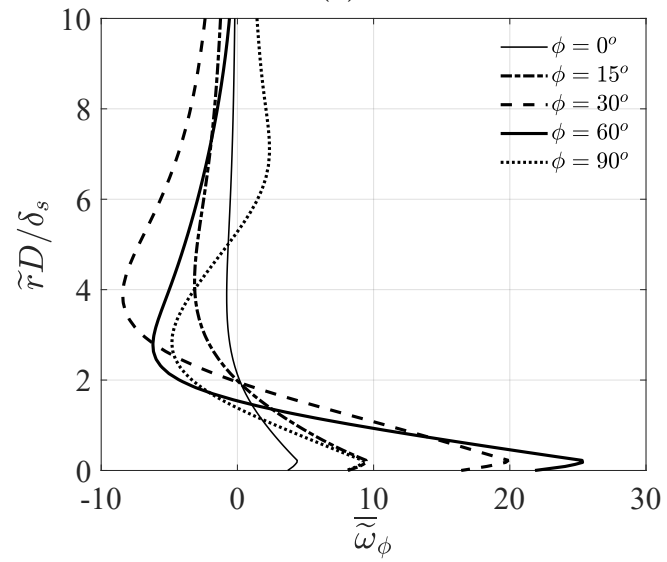

(c)

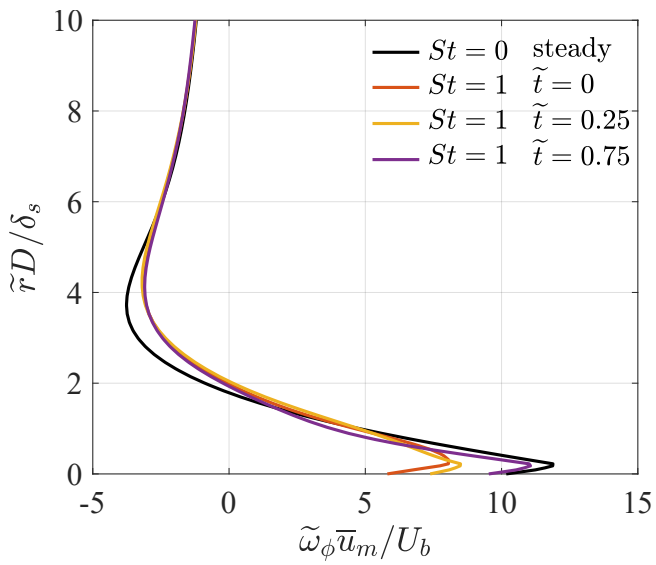

(b)

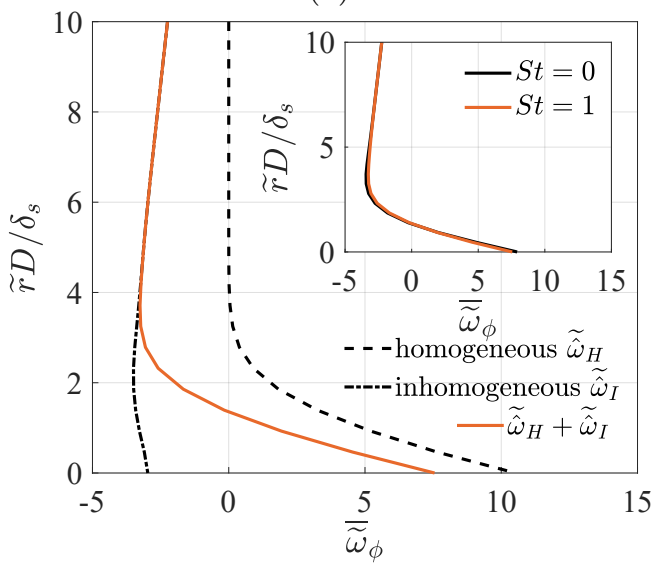

(d)

Figure 8. Variation of $\widetilde{\omega}_{\phi}$ with distance from the sidewall along line $\theta=90^{0}$ are shown. (a) Instantaneous $\widetilde{\omega}_{\phi}$ profiles for pulsatory inlet flow case $\left(R e_{b}=2000, S t=1\right.$ and $\left.R_{c} / D=1.5\right)$ are shown at a bend location $\phi=15^{0}$ and various times $(\widetilde{t}=0,0.25,0.5$, and 0.75). (b) Comparison of $\widetilde{\omega}_{\phi}$ between steady $\left(R e_{b}=2000\right.$ and $\left.S t=0\right)$ and pulsatory $\left(R e_{b}=2000\right.$ and $\left.S t=1\right)$ inlet flow conditions. (c) Time-averaged $\overline{\widetilde{\omega}}_{\phi}$ profiles are shown at various bend locations $\left(\phi=0,15,30,60\right.$ and $\left.90^{\circ}\right)$ based on numerical simulation results. $(d) \overline{\widetilde{\omega}}_{\phi}$ generation (based on linear model) from stretching $\left(\widetilde{\hat{\omega}}_{I}\right)$ and no-slip condition $\left(\widetilde{\hat{\omega}}_{H}\right)$ at $\phi=15^{0}$. The inset in $(d)$ shows the comparison of $\widetilde{\omega}_{\phi}$ between steady $\left(R e_{b}=2000\right.$ and $S t=0)$ and pulsatory $\left(R e_{b}=2000\right.$ and $\left.S t=1\right)$ inlet flow conditions.

\subsubsection{Streamwise vorticity}

Figure 8 shows the variation of streamwise vorticity $\widetilde{\omega}_{\phi}$ at various times $(\widetilde{t})$ and bend positions $(\phi)$. The $\widetilde{\omega}_{\phi}$ profiles (see figure $8 a$ ) in the lower sidewall bend region are negative in the core region (consistent with 3.15 ) because the source term is negative in the core and positive near the wall due to production via no-slip condition (see figure $7 b, c, d$ ). Due to the high-St, the upstream pipe flow consists of a steady distribution of $\widetilde{\omega}_{\theta}$ within the pipe core and an oscillatory boundary layer confined near the wall (see figure $6 a$ ). While $\widetilde{u}_{\phi}$ is unsteady, its profile is unchanged in the core region and simply moves backwards and forwards (see figure 4b,c), quite different from the low-St scenario. This results in the 


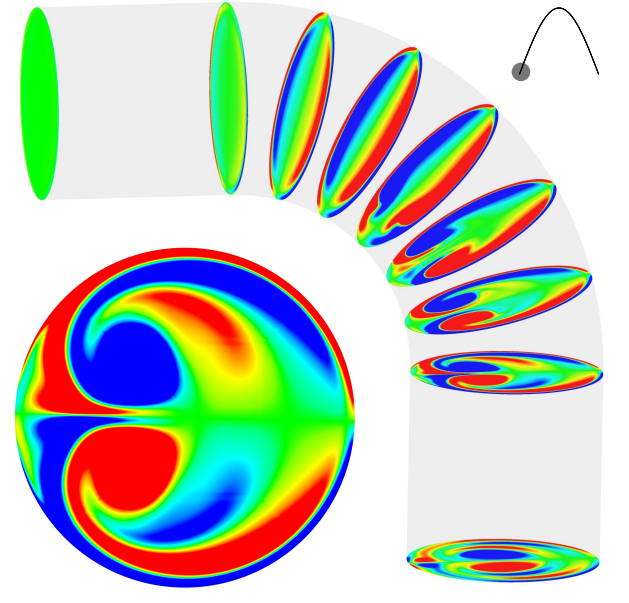

(a)

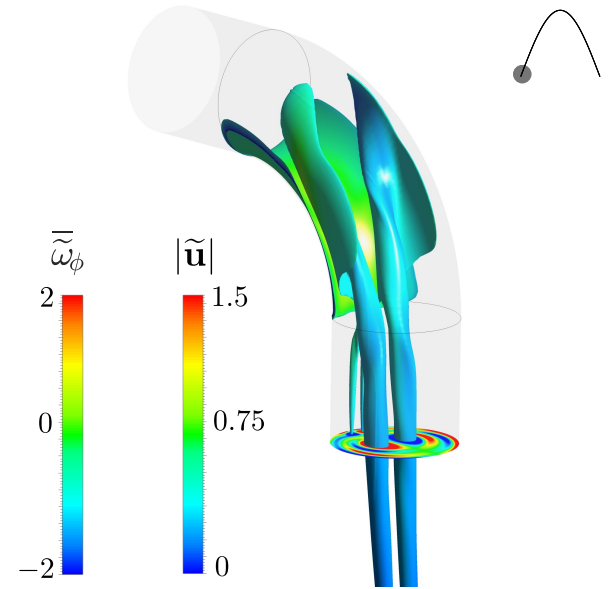

(b)

Figure 9. (a) Evolution of streamwise vorticity $\left(\widetilde{\widetilde{\omega}}_{\phi}\right)$ along pipe bend is shown with various cut-slices of flow, along with a snapshot at $\phi=90^{\circ}$. (b) Instantaneous vortical core structures are identified using an iso-surface of the second invariant (A 3$)(\widetilde{I I}=$ $5.8 \times 10^{-3}$ ) with the velocity magnitude $|\widetilde{\boldsymbol{u}}|$ plotted over iso-surfaces. The result is for a pulsatory flow with $R e_{b}=2000$ and $S t=1$ corresponding to $\widetilde{t}=0$.

source term from stretching $S$ that has a smaller in-cycle variation. The first observation is that $\widetilde{\omega}_{\phi}$ tends to be time-independent (steady) in the core region (see figure $8 a$ ). The second observation is that at high-St, $\tilde{\omega}_{\phi}$ has a close correspondence (in the core region) with the case $S t=0$ (see figure $8 b$ ). While Sumida et al. (1989) pointed out the similarity of the cross-flow streamlines, the range of $S t$ was limited to identify the relationship between the low- and moderately-high-St cases. The origin of weak dependence of $\tilde{\omega}_{\phi}$ on time and the similarity between $S t=0$ and $S t=1$ become clear when the production terms are compared (see figure $7 a$ ).

Figure $8(c)$ shows a comparison of $\overline{\widetilde{\omega}}_{\phi}$ profiles at various bend locations. Beyond $\phi=30^{\circ}$, the self-induced flow caused by the streamwise vorticity leads to rearrangement of streamwise vorticity (see figure $9 a$ ). Figure $8(d)$ shows the predictions by linear model highlighting the distinct contributions for the vorticity generated by stretching $\left(\widetilde{\hat{\omega}}_{I}\right)$ and from no-slip $\left(\widetilde{\hat{\omega}}_{H}\right)$ condition on the wall. The critical element is that shear in the streamwise flow enhances cross-stream diffusion, amplifying the streamwise vorticity $\left(\omega_{\phi}\right)$. This diffusive process is absent from the analysis of Hawthorne (1951). A comparison is shown between the $\overline{\widetilde{\omega}}_{\phi}$ prediction based on $S t=0$ and $S t=1$ (see inset in figure $8 d$ ). The comparison shows that the core flow distribution is identical, and the differences only occur near the wall. The linear model, based on small-curvature $\left(R_{c} / D \ll 1\right)$, provides a qualitative view of the numerical simulations, which are based on large-curvature $\left(R_{c} / D=1.5\right)$.

Figure $9(a)$ shows the cross-stream distribution of $\overline{\widetilde{\omega}}_{\phi}$ in several planes along bend length for a pulsatory inlet flow at $\widetilde{t}=0$. (Note: vorticity distribution at all $\tilde{t}=$ $0,0.25,0.5,0.7$ show similar characteristics as steady, and therefore only the results of $\tilde{t}=0$ are shown here). An iso-surface of the second invariant is plotted (see figure $9 b$ ) to differentiate the contribution of secondary motion from steady and an oscillatory boundary layer in the bend region. The transition from pure sidewall secondary vortex pair (in near bend region at $\phi=0^{0}$ to $15^{0}$ ) to the deformed secondary vortex pair in 


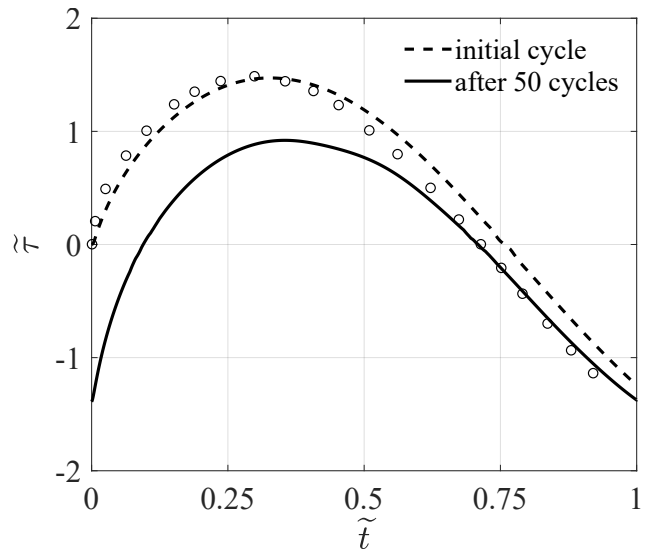

(a)

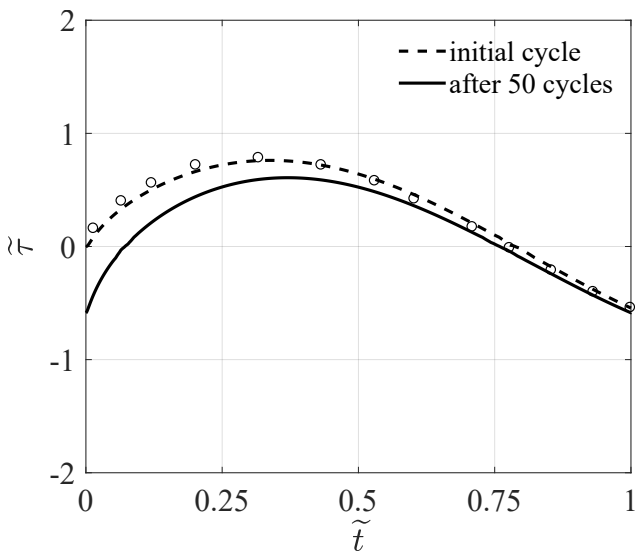

(b)

Figure 10. Variation of the dimensionless wall shear stress $\left(\widetilde{\tau}_{\phi r}\right)$ over one period is shown, contrasting the values on the $(a)$ inner and $(b)$ outer walls at a bend location $\phi=45^{0}$. The pulsatory parameters are $R e_{b}=2000$ and $S t=1$, and $R_{c} / D=1.5$. The shear stress in a strongly curved pipe calculated by Komai \& Tanishita (1997) is plotted with (o).

the inner bend (at $\phi=90^{\circ}$ ) is observed in both steady and pulsatory inlet flow. The Stokes region of a pulsatory inlet flow shows an additional near-wall coherent structure throughout the inner bend region, which is generated due to the strong negative velocity gradient at the impulsively-starting $(\widetilde{t}=0)$ and stopping phase $(\widetilde{t}=1)$.

\section{Dynamics of the flow field}

The bulk dynamics of the flow are driven by changes in pressure along the pipe and viscous stresses that act on the pipe wall; both elements are explored before we discuss the pipe bend forces.

\subsection{Viscous shear stress}

The viscous shear stress is proportional to the vorticity on the wall and provides an idea of the viscous component of the force. Figure 10 shows the variation of shear stress $\left(\widetilde{\tau}_{\phi r}\right)$ at a point in an inner (see figure 10a) and outer (see figure 10b) bend region over $\widetilde{t}=0$ and 1 . This is captured for the first cycle $\left(\Omega_{p} t / \pi\right)$ and 51 st cycle $\left(\Omega_{p} t / \pi-50\right)$ when the initial transient is lost. A comparison is made with the analysis of Komai \& Tanishita (1997) who uses a large-curvature pipe model to analyse flow adjustment over the initial cycle, which reasonably captures our observations of strong wall shear stress at the inner bend. The maximum shear stress at the outer bend is 35\% (during the first cycle) and $40 \%$ (during the 51st cycle) of the inner bend. Krishna et al. (2017) also showed a similar difference in the wall shear stress of $50 \%$ (ratio: outer to inner bend) during the first cycle for a square cross-sectional curved pipe.

\subsection{Pressure}

When $S t \geqslant S t_{c r}$, the pipe flow is alternately accelerated and decelerated along the pipe, and so the pressure is expected to vary linearly with distance (3.26). Figure 11(a) shows the pressure $\left(\widetilde{p}_{s}\right)$ variation over a pipe length along the inner-surface, center-line, and outer-surface, plotted against the centerline distance $(\widetilde{s})$, from the pipe inlet, confirming 


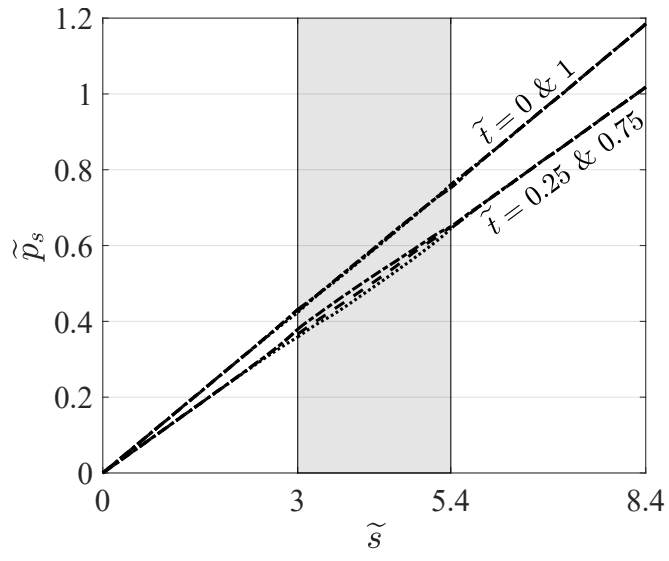

(a)

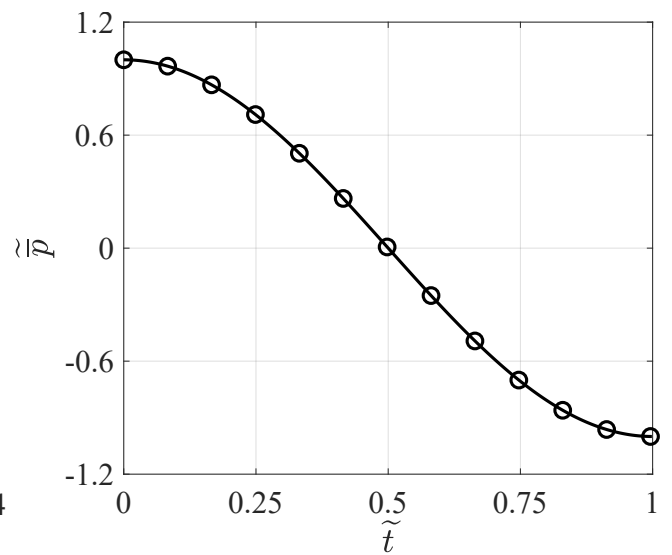

(b)

Figure 11. (a) Variation of the dimensionless centre, inner and outer wall pressure $\left(\widetilde{p}_{s}\right)$ with distance along the pipe from the inlet $(\widetilde{s}=s / D)$. The shaded region represents the bend region. ( $b$ ) The variation of cross-sectional averaged pressure $(\overline{\bar{p}})$ at a bend entry $\left(\phi=0^{0}\right)$ with time is shown over one cycle. The pulsatory parameters are $R e_{b}=2000$ and $S t=1$, and $R_{c} / D=1.5$.

the proportionality of $p$ to $\partial u / \partial t$ and $s$ (see 3.26). The shaded region represents the extent of the bend region. The pressure gradient changes sign during one cycle, but the non-dimensional form in figure $11(a)$ is positive. The small deviation from the linear variation within the bend region during an accelerating $(\widetilde{t}=0.25)$ and decelerating $(\widetilde{t}=0.75)$ phases is due to viscous effects and flow curvature. The parametric variation of pressure distribution in a curved pipe at various $S t$ is discussed in the figure 14 (see Appendix D). The cross-sectional averaged pressure $\bar{p}$ at a bend inlet is plotted over one cycle in figure $11(b)$ and is characterized by a discontinuity between $\tilde{t}=0$ and 1 due to the impulsive acceleration changing sign. The change in sign from positive to negative has an important implication for the potential for cavitation. This aspect was analysed by Wachel et al. (1989).

\subsection{Forces}

Figure $12(a, b)$ shows a decomposition of the bend force components $\left(\widetilde{F}_{x}\right.$ and $\left.\widetilde{F}_{y}\right)$ into the various terms shown in (3.18) and compared with the one-dimensional analytical model (3.24). This shows the relative calculation of pressure at the inlet/outlet of bend $\left(\bar{p}_{b i}\right.$ and $\left.\bar{p}_{b o}\right)$, flow acceleration $\left(\rho \int_{V} \frac{\partial \boldsymbol{u}}{\partial t} \mathrm{~d} V\right)$, streamwise momentum flux changes $\left(\rho \int_{S_{b i}} \overline{u_{\phi}^{2}} \mathrm{~d} A\right.$ and $\left.\rho \int_{S_{b o}} \overline{v^{\prime 2}} \mathrm{~d} A\right)$, crosswise momentum flux changes $\left(\rho \int_{S_{b o}} \overline{u_{\phi} v^{\prime}} \mathrm{d} A\right.$ and $\left.\rho \int_{S_{b i}} \overline{u_{\phi} v^{\prime}} \mathrm{d} A\right)$ to the total bend force. These bend loading components are dominated by the unsteady pressure field for $S t \gg S t_{c r}$. The agreement between predictions and computations give a strong justification that the key physics are encapsulated in the simple one-dimensional model (3.24). The analysis indicates that the force components depend on the bend length $\left(R_{c} \pi / 2\right)$ and pipe exit length $\left(L_{d}\right)$ since this largely determine the pressure in the vicinity of bend. The model also predicts an asymmetry between $\widetilde{F}_{x}$ and $\widetilde{F}_{y}$ (see figure $12 c$ ), especially near $\widetilde{t}=0$ and $\widetilde{t}=1$. This difference is due to $R_{c} \pi / 2$ and $L_{d}$.

The influence of curvature $\left(R_{c} / D\right)$ on $\widetilde{F}_{x}$ is shown in figure $12(d)$. The variation in $\widetilde{F}_{x}$ is due to the enhancement or dehancement of bend inlet pressure $\left(p_{b i}=\rho \frac{\partial u_{m}}{\partial t}\left(L_{d}+\pi \frac{R_{c}}{2}\right)\right)$ 


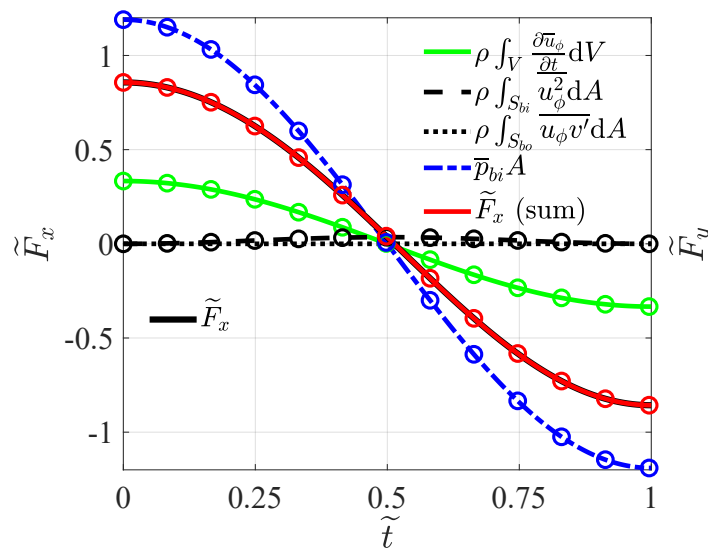

(a)

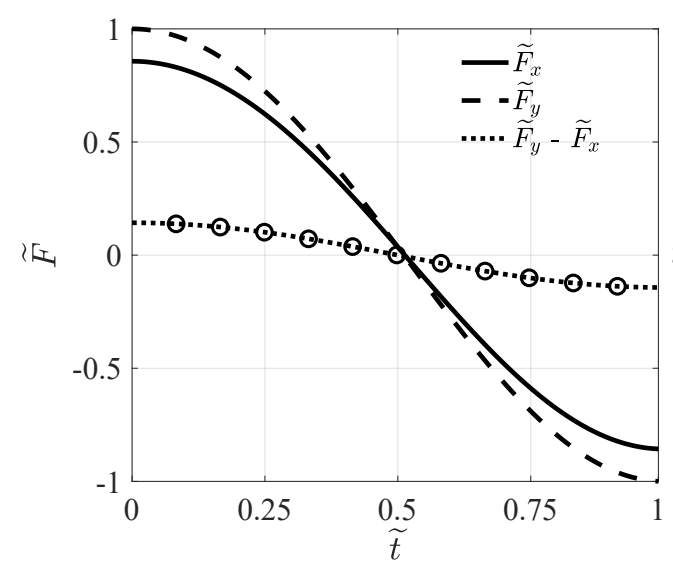

(c)

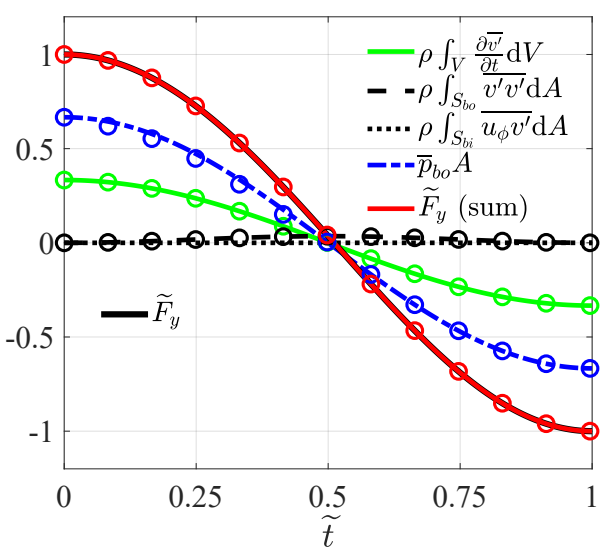

(b)

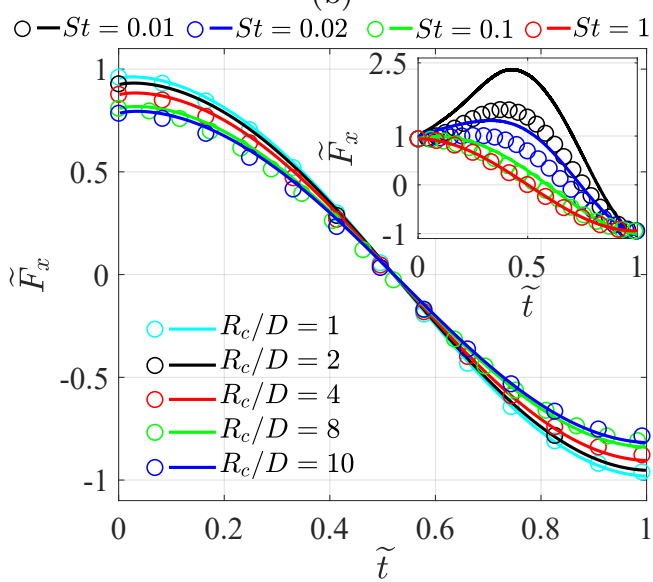

(d)

Figure 12. The dimensionless components of bend force $\widetilde{F}_{x}$ and $\widetilde{F}_{y}$ are plotted as a function of time. $(a, b) \widetilde{F}_{x}$ and $\widetilde{F}_{y}$ are decomposed into integral components and evaluated numerically (3.18; solid lines) and estimated analytically (3.24; circular markers). [Note: (i) the components $\rho \int_{S_{b i}} \overline{u_{\phi}^{2}} \mathrm{~d} A$ and $\rho \int_{S_{b o}} \overline{u_{\phi} v^{\prime}} \mathrm{d} A$ coincide to $\widetilde{F}_{x}=0$ axis, and the components $\rho \int_{S_{b o}} \overline{v^{\prime} v^{\prime}} \mathrm{d} A$ and $\rho \int_{S_{b i}} \overline{u_{\phi} v^{\prime}} \mathrm{d} A$ coincide to $\widetilde{F}_{y}=0$ axis, (ii) the plots of $\widetilde{F}_{x}$ (sum) and $\widetilde{F}_{y}$ (sum) with red solid lines are the summation of all the components, and the plots of $\widetilde{F}_{x}$ and $\widetilde{F}_{y}$ with black solid lines are the total force computed directly from FLUENT - both red and black solid lines collapse, (iii) the results are for a pulsatory inlet flow with $R e_{b}=2000, S t=1$, and $R_{c} / D=1.5$. (c) Comparison of $\widetilde{F}_{x}$ and $\widetilde{F}_{y}$ is shown in terms of force difference estimated numerically (solid, dashed and dotted lines) and analytically (3.29). (d) Variation of $\widetilde{F}_{x}$ at $R e_{b}=2000$ and $S t=1$ with respect to $R_{c} / D$ is highlighted. Inset in $(d)$ describes the discrimination between flow-accelerationcontrolled $\left(S t \gg S t_{c r}\right)$ and momentum-flux-controlled force behaviour at $R e_{b}=2000$ and $R_{c} / D=1.5$, and the numerical results at $S t=0.01,0.02,0.1$, and 1 are compared with the analytical predictions (3.27). 
and bend volume flow acceleration $\left(\rho \int_{V} \frac{\partial \boldsymbol{u}}{\partial t} \mathrm{~d} V=\rho \frac{\partial u_{m}}{\partial t} R_{c} A\right)$. The sensitivity of $\widetilde{F}_{x}$ to $S t$ (with $R e_{b}=2000$ and $R_{c} / D=1.5$ ) is shown as an inset in figure $12(d)$. At high-St (flow-acceleration controlled zone: $S t \geqslant S t_{c r}$ and $R e_{b} \gg 1$ ), it shows good agreement between the predictions (3.27) and numerical results. While the slight disagreement at low-St (momentum-flux controlled zone: $S t \ll S t_{c r}$ and $R e_{b} \gg 1$ ) is attributed to viscous diffusion. These loading regimes (flow-acceleration and momentum-flux controlled zone) are highlighted in the figure 1 .

\section{Conclusions}

The main contribution of this study is a general analysis at high-St of the force loading and secondary flow in a curved pipe. A detailed one-dimensional force analysis shows an asymmetry in the pipe bend force components. The total bend force component has three contributions: the pressure force at the inlet/outlet of the bend, flow acceleration, and momentum flux changes (3.18). The essential character of the model has been successfully compared against numerical simulation over a range of $S t$ and $R_{c} / D$, spanning the quasi-steady flow regime $\left(S t \ll S t_{c r}\right)$ where the pressure force dominates the force due to change in momentum flux to the unsteady regime $\left(S t \geqslant S t_{c r}\right)$, where the force is dominated by pressure force due to flow-acceleration.

The study highlights that the spatial distribution of the streamwise vorticity in the steady inlet flow case is similar to the high-St limit. This occurs because the inlet flow upstream of the bend tends to a parabolic profile (with an oscillatory boundary layer and offset near the wall), leading to a streamwise vorticity source, which is similar in both cases. The difference that occurs can be explained simply from the production terms and a linear model of the streamwise vorticity.

Although an idealised volume flux has been assumed for the pulsatory inlet flow, corresponding to one typical of reciprocating pumps, the analysis developed for the force and secondary flow are much more general. This is because all pulsatory flows ultimately tend to a similar asymptotic form (see (B 2)) adopted in this paper as an inlet condition for the numerical simulations. This means that the models are of general significance to a wide variety of pump profiles operating at high-St.

Finally, in practice, fluid compressibility is important, and this leads to additional physical processes not present in our analysis of an incompressible fluid. This will be dealt with in our future work.

\section{Acknowledgement}

The authors acknowledge the use of the Grace@UCL, Legion@UCL, Kathleen@UCL, and Myriad@UCL High-Performance Computing Facility and associated support services. This work is supported by the Engineering and Physical Science Research Council (EPSRC) and British Petroleum (BP).

\section{Appendix A. Diagnostic Tools}

\section{A.1. Coordinate transformation}

The toroidal coordinate frame is a natural system to analyse data in the pipe bend region since it distinguishes between the flow perpendicular to the pipe cross-section $\left(u_{\phi}\right)$ and the secondary flow parallel to the pipe $\left(u_{r}, u_{\theta}\right)$ (see figure $2 c$ ). The velocity and velocity gradient fields in the toroidal range of $0 \leqslant \phi \leqslant 90^{0}$ were mapped from 
the Cartesian coordinate system $\left(\boldsymbol{u}=(u, v, w)^{T}, \boldsymbol{\nabla} \boldsymbol{u}\right)$ to the toroidal coordinate system $\left(\boldsymbol{u}^{\prime}=\left(u_{r}, u_{\theta}, u_{\phi}\right)^{T}, \nabla \boldsymbol{u}^{\prime}\right)$ by the rotation matrix $\boldsymbol{R}$ when

$$
\boldsymbol{u}^{\prime}=\boldsymbol{R} \cdot \boldsymbol{u}, \quad \nabla \boldsymbol{u}^{\prime}=\boldsymbol{R} \cdot \nabla \boldsymbol{u} \cdot \boldsymbol{R}^{\boldsymbol{T}}, \quad \boldsymbol{R}=\underbrace{\left[\begin{array}{ccc}
0 & \cos \theta & \sin \theta \\
0 & -\sin \theta & \cos \theta \\
1 & 0 & 0
\end{array}\right]}_{\boldsymbol{R}_{\boldsymbol{x}^{\prime}}} \underbrace{\left[\begin{array}{ccc}
\cos \phi & -\sin \phi & 0 \\
\sin \phi & \cos \phi & 0 \\
0 & 0 & 1
\end{array}\right]}_{\boldsymbol{R}_{\boldsymbol{z}}} .
$$

$u_{r}, u_{\phi}$, and $u_{\theta}$ are the radial, streamwise, and azimuthal velocity components, respectively. Use $\phi$ as $90^{\circ}-\phi$ in ANSYS CFDPost, and use a gradient filter in Paraview to estimate the transformed velocity and velocity gradients.

\section{A.2. Vortex identification}

The vorticity at a point is defined as, $\boldsymbol{\omega}=\boldsymbol{\nabla} \times \boldsymbol{u}$. Since the largest magnitude of $\boldsymbol{\omega}$ is on the pipe wall surface, it is difficult to use the magnitude $|\boldsymbol{\omega}|$ to discriminate vortical structures within the internal flows. Instead, the second invariant of velocity gradient tensor (Hunt et al. 1988), defined as

$$
I I=\frac{1}{2}\left(\|\Omega\|^{2}-\|S\|^{2}\right),
$$

where, $\boldsymbol{\Omega}=\frac{1}{2}\left(\nabla \boldsymbol{u}-(\nabla \boldsymbol{u})^{T}\right)$ and $\boldsymbol{S}=\frac{1}{2}\left(\nabla \boldsymbol{u}+(\nabla \boldsymbol{u})^{T}\right)$, is a more useful measure to identify vortices. This measure can distinguish between linear shear $(I I=0)$, which dominates the vorticity field, a vortical field $(I I>0)$ and straining region $(I I<0)$.

The pipe coordinates, streamwise velocity $\left(u_{\phi}\right)$, streamwise vorticity $\left(\omega_{\phi}\right)$, azimuthal vorticity $\left(\omega_{\theta}\right)$, and second invariant $(I I)$ are non-dimensionalised by internal scaling parameters as

$$
\begin{gathered}
\widetilde{y}^{\prime}=\frac{y^{\prime}-R_{c}}{D}, \quad \widetilde{z}^{\prime}=\frac{z^{\prime}}{D}, \quad \widetilde{r}=\frac{D-2 r}{2 D}, \quad \widetilde{u}_{\phi}=\frac{u_{\phi}}{U_{b}}, \\
\widetilde{\omega}_{\phi}=\frac{\omega_{\phi} D}{U_{b}}, \quad \widetilde{\omega}_{\theta}=\frac{\omega_{\theta} D}{U_{b}}, \quad \widetilde{I I}=\frac{I I D^{2}}{U_{b}{ }^{2}} .
\end{gathered}
$$

\section{A.3. Forces}

The total force acting on the pipe bend surface $S_{b w}$ (shown in figure $2 c$ ) is defined as

$$
\boldsymbol{F}_{p}=\int_{S_{b w}}(-p \boldsymbol{I}+\boldsymbol{\tau}) \cdot \hat{\boldsymbol{n}} \mathrm{dS},
$$

where $\boldsymbol{I}$ is the identity tensor, $\hat{\boldsymbol{n}}$ is the unit normal to the surface pointing out of the fluid domain (Klettner et al. 2016). The dimensionless form of spatial pressure field, temporal pressure, and shear stress field can be expressed as

$$
\widetilde{p}_{s}=\frac{p_{i}-p}{\rho L_{t} \frac{\partial u_{m}}{\partial t}}, \quad \tilde{p}=\frac{p}{\rho U_{b} \Omega_{p} L_{t}}, \quad \widetilde{\tau}_{\phi r}=\frac{\tau_{\phi r} \delta_{s}}{U_{b}},
$$

respectively, where $p_{i}$ is the centerline pressure at the pipe inlet. Also, the force components can be written in non-dimensionalised form as

$$
\widetilde{F}_{x}=\frac{F_{x}}{\rho A \Omega_{p} U_{b}\left(L_{d}+R_{c}\right)}, \quad \widetilde{F}_{y}=\frac{F_{y}}{\rho A \Omega_{p} U_{b}\left(L_{d}+R_{c}\right)} .
$$




\section{Appendix B. Uniform pipe flow}

With the given volumetric flux at the pipe inlet, Das \& Arakeri (2000) provides a series of analytical solution for various flow rate by solving one-dimensional Navier-Stokes equation using Laplace transform. We have adopted the similar approach and extended the solution to a general arbitrary piston flow (also done by Muntges \& Majdalani (2002) and Venudas \& Manu (2018)), written as,

$$
u_{m}(t)=u_{0}+\sum_{n=1}^{\infty} u_{c n} \cos n \Omega t+\sum_{n=1}^{\infty} u_{s n} \sin n \Omega t,
$$

where $u_{m}(t)$ is cross-sectional averaged velocity (or bulk velocity or flow rate), $u_{0}=\bar{u}_{m}$ is the (mean) steady velocity component; $u_{c n}=a_{n} U_{m}$ and $u_{s n}=b_{n} U_{m}$ are cosine and sine velocity components, respectively; and $U_{m}$ is the maximum of cosine and sine velocity components. The bulk velocity of a specific case can be estimated by substituting the Fourier coefficients $\left(a_{n}\right.$ and $\left.b_{n}\right)$. On applying Laplace transform to 3.1 and 3.2 and estimating the inverse Laplace transform using Residue method the solution for radial velocity becomes

$$
u(r, t)=u_{1}(r, t)+u_{2}(r, t)+u_{3}(r, t)
$$

where

$$
\begin{gathered}
u_{1}(r, t)=2 \bar{u}_{m}\left(1-c^{2}\right), \\
u_{2}(r, t)=\sum_{n=1}^{\infty}\left[\frac{-u_{c n}}{2}\left(\frac{J_{0}\left(\gamma_{a}\right)-J_{0}\left(c \gamma_{a}\right)}{J_{2}\left(\gamma_{a}\right)} e^{i n \Omega t}+\frac{J_{0}\left(\gamma_{b}\right)-J_{0}\left(c \gamma_{b}\right)}{J_{2}\left(\gamma_{b}\right)} e^{-i n \Omega t}\right)\right. \\
\left.+\sum_{m=1}^{\infty} \frac{16 u_{c n} \nu^{2}}{16 \gamma_{m}^{4} \nu^{2}+D^{4} n^{2} \Omega^{2}}\left(\frac{2 \gamma_{m}^{3}\left(J_{0}\left(\gamma_{m}\right)-J_{0}\left(c \gamma_{m}\right)\right)}{J_{1}\left(\gamma_{m}\right)}\right) e^{\left(-\gamma_{m}^{2} 4 \nu / D^{2}\right) t}\right], \\
u_{3}(r, t)=\sum_{n=1}^{\infty}\left[\frac{i u_{s n}}{2}\left(\frac{J_{0}\left(\gamma_{a}\right)-J_{0}\left(c \gamma_{a}\right)}{J_{2}\left(\gamma_{a}\right)} e^{i n \Omega t}-\frac{J_{0}\left(\gamma_{b}\right)-J_{0}\left(c \gamma_{b}\right)}{J_{2}\left(\gamma_{b}\right)} e^{-i n \Omega t}\right)\right. \\
\left.+\sum_{m=1}^{\infty} \frac{4 u_{s n} D^{2} \nu n \Omega}{16 \gamma_{m}^{4} \nu^{2}+D^{4} n^{2} \Omega^{2}}\left(\frac{2 \gamma_{m}\left(J_{0}\left(\gamma_{m}\right)-J_{0}\left(c \gamma_{m}\right)\right)}{J_{1}\left(\gamma_{m}\right)}\right) e^{\left(-\gamma_{m}^{2} 4 \nu / D^{2}\right) t}\right]
\end{gathered}
$$

where $c=2 r / D ;-i \gamma_{a}=\frac{D}{2} \sqrt{\frac{i n \Omega}{\nu}} ;-i \gamma_{b}=\frac{D}{2} \sqrt{\frac{-i n \Omega}{\nu}} ; J_{0}, J_{1}$ and $J_{2}$ are the Bessel function of first kind for zero, first, and second order, respectively; and $\gamma_{m}$ (where $m=$ $1,2,3, \ldots, \infty)$ are zeros of $J_{2}$.

\section{B.1. Special cases}

The solution (B 2) can be estimated for the bulk velocity of any specific flow rate by substituting the Fourier coefficients $\left(a_{n}\right.$ and $\left.b_{n}\right)$ :

(i) for steady flow $\left(u_{0}=\bar{u}_{m}, a_{n}=0, u_{c n}=0, b_{n}=0, u_{s n}=0\right.$ and $\left.n=0\right)$,

$$
u_{m}(t)=\bar{u}_{m}, \quad u(r, t)=u_{1}(r, t),
$$

(ii) for zero-mean oscillatory flow (or pure sinusoidal flow, $u_{0}=0, a_{n}=0, u_{c n}=0, b_{n}=$ 1, $u_{s n}=U_{m}$ and $n=1$ ),

$$
u_{m}(t)=U_{m} \sin \Omega t, \quad u(r, t)=u_{3}(r, t),
$$


High-Strouhal-number pulsatory flow in a curved pipe

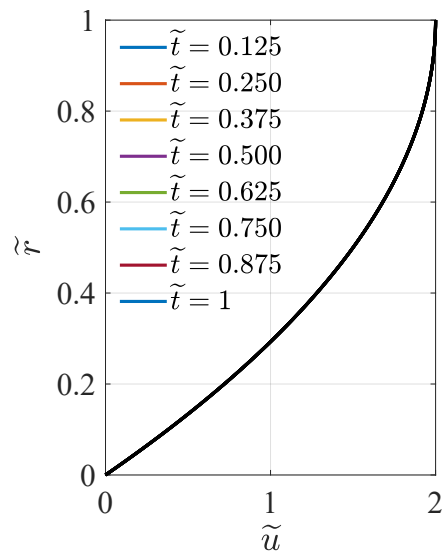

(a)

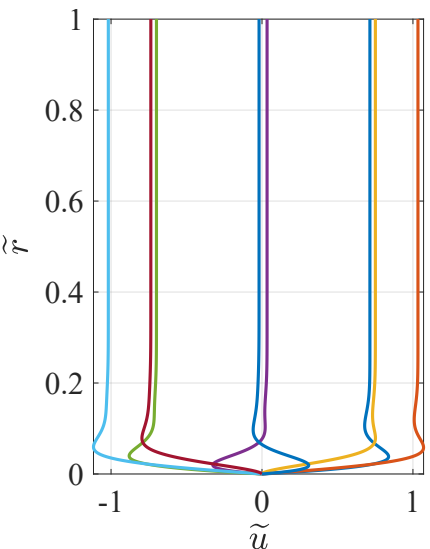

(b)

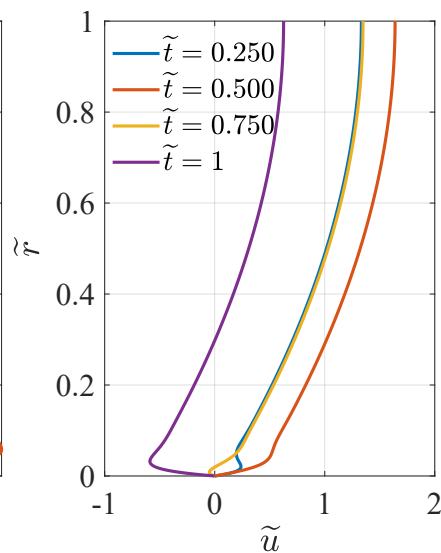

(c)

Figure 13. Comparison of the nature of an inlet condition, generated analytically in an infinitely long pipe (B 2) along $\widetilde{r}\left(=\left.\widetilde{r}\right|_{\theta=0}\right)=1-2 r / D$ in an upstream straight pipe, for a (a) steady flow $\left(R e_{b}=2000\right)$ (B 6), (b) zero-mean oscillatory flow $\left(R e_{b}=2000, D / \delta_{s}=\right.$ 45) (B 7), and (c) an impulsively-accelerated flow $\left(R e_{b}=2000, D / \delta_{s}=45\right)$ (B 9).

(iii) for pulsatory flow of mean-oscillation $\left(u_{0}=\bar{u}_{m}, a_{n}=0, u_{c n}=0, b_{n}=1, u_{s n}=U_{m}\right.$ and $n=1$ ),

$$
u_{m}(t)=\bar{u}_{m}+U_{m} \sin \Omega t, \quad u(r, t)=u_{1}(r, t)+u_{3}(t),
$$

(iv) for pulsatory flow of positive pulse (2.2) $\left(u_{0}=\bar{u}_{m}, u_{c n}=a_{n} U_{m}, b_{n}=0, u_{s n}=0\right)$,

$$
u_{m}(t)=\bar{u}_{m}+U_{m} \sum_{n=1}^{\infty} a_{n} \cos n \Omega t, \quad u(r, t)=u_{1}(r, t)+u_{2}(r, t),
$$

where $\Omega=2 \Omega_{p}$ and

$$
a_{n}=\frac{4}{\pi\left(1-4 n^{2}\right)}
$$

for an impulsively-accelerated flow (2.2).

\section{B.2. Comment on the nature of an inlet condition}

It is worth understanding the nature of the inlet profile, which influences the secondary flow formation and forces in a curved pipe. In the case of impulsively-accelerated flow (non-zero oscillatory flow) (B9) at high Strouhal number $(S t=1)$ and $D / \delta_{s}=45$, the rms is comparable to the mean. So, the effect of both steady (see figure 13a) and zero-mean oscillatory (see figure 13b) flows are present in the velocity profile (see figure $13 c$ ). This contrasts with the zero-mean oscillatory flow and non-zero oscillatory flow (when the rms is larger than the mean component), where the profiles are approximately parabolic at $D / \delta_{s} \ll 0$ and flat at $D / \delta_{s} \gg 0$. The phase difference between velocity and pressure gradient of a high pulsatory flow $\left(D / \delta_{s} \gg 0\right)$ is $90^{0}$ due to the dominance of flow acceleration over viscous diffusion (Uchida 1956; Haddad et al. 2010); which leads to jump-discontinuities in the flow acceleration in an impulsively-accelerated pipe flow $(2.2)$. 


\section{B.3. Numerical implementation of an inlet condition}

A uniform flow analytical solution for the unsteady Navier-Stokes equation in terms of velocity profiles is prescribed at the upstream pipe inlet to simulate the physical condition (infinitely long upstream pipe). The execute-commands method in the FLUENT solver is used to read and interpolate the analytically obtained velocity profiles at the pipe inlet at each time-step for transient simulations. Discontinuity in flow acceleration (due to the flow being expressed as Fourier series in time) leads to a small unphysical oscillation (Gibbs phenomenon (Ray et al. 2005)) This is removed using Lanczos' sigma factor (as used by Ray et al. (2005)).

\section{Appendix C. Secondary flow analysis}

To analyse the cross-stream flow, we write $\widetilde{\omega}_{\phi}=U_{b} / D \widetilde{\hat{\omega}}(r, \phi) \sin \theta$. On substitution into (3.13), the non-dimensional form of (3.13) can be written as

$$
\frac{\partial \tilde{\hat{\omega}}}{\partial \widetilde{t}}+\widetilde{u}_{\phi} \frac{\partial \tilde{\hat{\omega}}}{\partial \widetilde{s}}=\frac{\gamma D}{R_{c}} \widetilde{\omega}_{\theta} \widetilde{u}_{\phi}+\frac{1}{R e_{b}}\left(\frac{\partial^{2}}{\partial s^{2}}+\frac{\partial^{2}}{\partial r^{2}}+\frac{1}{r} \frac{\partial}{\partial r}-\frac{1}{r^{2}}\right) \tilde{\hat{\omega}} .
$$

The streamwise vorticity $\left(\widetilde{\omega}_{\phi}\right)$ generates a cross-stream flow which can be expressed in terms of stream function $f$ as $\widetilde{u}_{r}=f \cos \theta / \widetilde{r}, \widetilde{u}_{\theta}=-f^{\prime} \sin \theta$, and satisfies,

$$
f^{\prime \prime}+\frac{1}{\hat{r}} f^{\prime}-\frac{1}{\hat{r}^{2}} f=-\tilde{\hat{\omega}} .
$$

This is solved subject to a kinematic condition $f(0)=f(1 / 2)=0$. The no-slip condition leads to the production of vorticity on the wall. This is imposed by writing $\widetilde{\hat{\omega}}=\widetilde{\hat{\omega}}_{H}+\widetilde{\hat{\omega}}_{I}$ and solving separately the vorticity contribution due to stretching $\left(\hat{\omega}_{I}\right)$ and the part due to the no-slip condition $\left(\hat{\omega}_{H}\right)$. The no-slip condition is achieved by updating the wall value of $\hat{\omega}_{H}$. The boundary condition for $\hat{\omega}_{I}$ are zero on the centerline $\left(\tilde{r}^{*}=0\right)$ with a zero gradient on the wall $\left(\widetilde{r^{*}}=1 / 2\right)$.

(C 1) is solved numerically with a finite volume formulation (a bespoke solver in $\mathrm{C}++$ ) linked to OpenFOAM2.4. A rectangular domain is used with the forcing applied at the pipe bend $(\phi>0)$. The boundary condition for $\hat{\omega}_{H}$ was adjusted every time-step.

\section{Appendix D. Pressure variation along the pipe length}

The pressure varies along a straight pipe at high- $R e_{b}$ due to viscous effects and flow acceleration, and varies long the pipe bend due to (mainly) inertial effects. For steady inlet flows $(S t=0)$, the pressure variation within the pipe bend is large compared to the pressure difference between the pipe bend exit and inlet due to the centrifugal effect (see figure 14a). The pressure characteristics in a steady pipe flow are similar to the experimental results described in figure 10 of Vester et al. (2016). As St increases $(S t=0.02,0.1,1)$, the pressure gradient is dominated by flow acceleration and varies linearly with distance in the straight pipe sections (see figure 14b, $c$ and $12 a$, respectively).

\section{REFERENCES}

Agrawal, Y., Talbot, L. \& Gong, K. 1978 Laser anemometer study of flow development in curved circular pipes. J. Fluid Mech. 85, 497-518.

Bertelsen, A. F. 1975 An experimental investigation of low reynolds number secondary streaming effects associated with an oscillating viscous flow in a curved pipe. J. Fluid Mech. 70, 519-527. 


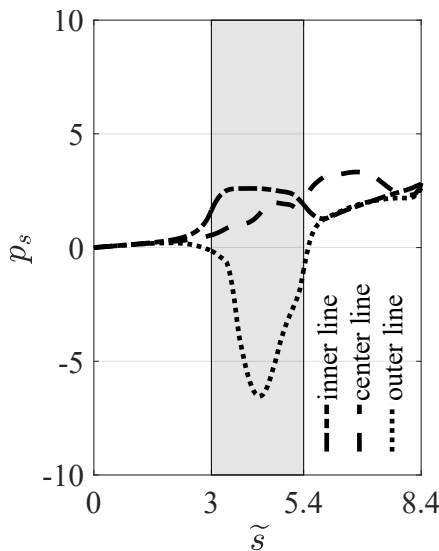

(a)

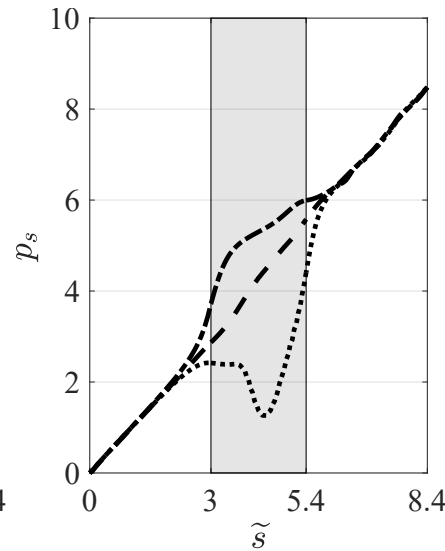

(b)

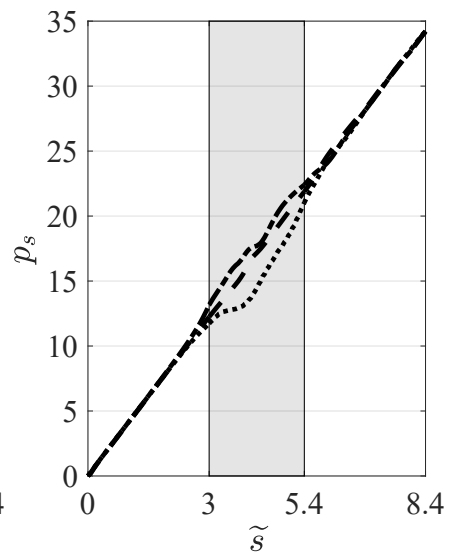

(c)

Figure 14. Comparison of pressure variation $p_{s}\left(=p_{i}-p\right)$ along the length of pipe $(\widetilde{s}=$ $s / D)$ between $(a)$ steady and $(b, c)$ unsteady pipe flows at $\widetilde{t}=0.25$ for $(b) S t=0.02$ and (c) $S t=0.1$. The pressure characteristics in a steady pipe flows is also shown in figure 10 of Vester et al. (2016).

Boiron, O., Deplano, V. \& Pelissier, R. 2007 Experimental and numerical studies on the starting effect on the secondary flow in a bend. J. Fluid Mech. 574, 109-129.

Cox, C., NajJari, M. R. \& Plesniak, M. W. 2019 Three-dimensional vortical structures and wall shear stress in a curved artery model. Phy. Fluids 31 (12).

DAs, D. \& ArAKeri, J. H. 1998 Transition of unsteady velocity profiles with reverse flow. J. Fluid Mech. 374, 251-283.

DAs, D. \& Arakeri, J. H. 2000 Unsteady laminar duct flow with a given volume flow rate variation. J. Appl. Mech. 67 (2), 274-281.

Das, S. P., Srinivasan, U. \& Arakeri, J. H. 2016 Instabilities in unsteady boundary layers with reverse flow. Eur. J. Mech.-B/Fluids 55, 49-62.

Dean, W. R. 1927 XVI. Note on the motion of fluid in a curved pipe. Lond. Edinb. Dublin Phil. Mag. J. Sci. 4 (20), 208-223.

Dean, W. R. 1928 LXXII. The stream - line motion of fluid in a curved pipe. Lond. Edinb. Dublin Phil. Mag. J. Sci. 5 (30), 673-695.

Einstein, A. 1926 The cause of the formation of meanders in the courses of rivers and of the so-called Baer's law. Die Naturwissenschaften 14, 223-224.

Ghadirian, A. \& Bredmose, H. 2019 Pressure impulse theory for a slamming wave on a vertical circular cylinder. J. Fluid Mech. 867, 1-14.

Haddad, K., Ertunç, Ö., Mishra, M. \& Delgado, A. 2010 Pulsating laminar fully developed channel and pipe flows. Phys. Rev. E 81 (1), 016303.

Hawthorne, W. R. 1951 Secondary circulation in fluid flow. Proc. R. Soc. Lond. A. 206 (1086), $374-387$.

Hellstrom, F. \& FuChs, L. 2007 Numerical computations of steady and unsteady flow in bended pipes. In 37th AIAA Fluid Dyn. Conf.

Hou, D. Q., Tijsseling, A. S. \& Bozkus, Z. 2014 Dynamic force on an elbow caused by a traveling liquid slug. J. Press. Vess. Tech. 136 (3).

Hunt, J. C. R. 1973 A theory of turbulent flow round two-dimensional bluff bodies. J. Fluid Mech. 61, 625-706.

Hunt, J. C. R, Abell, C. J., Peterka, J. A. \& Woo, H. 1978 Kinematical studies of the flows around free or surface-mounted obstacles; applying topology to flow visualization. J. Fluid Mech. 86 (1), 179-200.

Hunt, J. C. R., Wray, A. A. \& Moin, P. 1988 Eddies, streams, and convergence zones in turbulent flows. Center for Turbulence Research Report CTR-S88 p. 193. 
InOuE, O. 1981 Mrs criterion for flow separation over moving walls. AIAA J. 19 (9), 1108-1111. Inthavong, K. 2019 A unifying correlation for laminar particle deposition in 90-degree pipe bends. Pow. Tech. 345, 99-110.

Jarrahi, M., Castelain, C. \& Peerhossaini, H. 2011 Secondary flow patterns and mixing in laminar pulsating flow through a curved pipe. Exps. Fluids 50 (6), 1539-1558.

Kalpakli, A., Örlü, R., Tillmark, N. \& Alfredsson, P. H. 2010 Experimental investigation on the effect of pulsations on turbulent flow through a 90 degrees pipe bend. Proc. 3rd Intl. Conf. on Jets, Wakes and Separated Flows, Cincinnati, Ohio, USA .

KARTiK, V. B. \& Michael, W. P. 2018 Insights on arterial secondary flow structures and vortex dynamics gained using the MRV technique. Intl. J. Heat Fluid Flow 73, 143-153.

Klettner, C. A., Eames, I., Semsarzadeh, S. \& Nicolle, A. 2016 The effect of a uniform through-surface flow on a cylinder and sphere. J. Fluid Mech. 793, 798-839.

Komai, Yutaka \& TAnishita, KaZuo 1997 Fully developed intermittent flow in a curved tube. J. Fluid Mech. 347, 263-287.

Krishna, C. V., Gundiah, N. \& Arakeri, J. H. 2017 Separations and secondary structures due to unsteady flow in a curved pipe. J. Fluid Mech. 815, 26-59.

Lighthill, M. J. 1956 Drift. J. Fluid Mech. 1, 31-53.

Lyne, W. H. 1971 Unsteady viscous flow in a curved pipe. J. Fluid Mech. 45, 13-32.

Mahmoudi Zarandi, M. 2000 Steady and pulsatile flow in curved vessels. PhD thesis, California Institute of Technology.

ManRING, N. D. 2000 The discharge flow ripple of an axial-piston swash-plate type hydrostatic pump. J. Dyn. Sys., Meas. and Cont., Trans. ASME 122, 263-268.

MilLeR, J. E. 1987 The reciprocating pump: theory, design, and use. Wiley.

Miller, J. E. 1988 Characteristics of the reciprocating pump. In Proc. 5th Intl. Pump Users Symp.

Muntges, D. \& Majdalani, J. 2002 Pulsatory channel flow for an arbitrary volumetric flowrate. In 32nd AIAA Fluid Dynamics Conference and Exhibit, p. 2856.

NajJari, M. R., Cox, C. \& Plesniak, M. 2019 Formation and interaction of multiple secondary flow vortical structures in a curved pipe: transient and oscillatory flows. $J$. Fluid Mech. 876, 481-526.

NAJJARI, M. R. \& Plesniak, M. W. 2016 Evolution of vortical structures in a curved artery model with non-newtonian blood-analog fluid under pulsatile inflow conditions. Exp. Fluids $\mathbf{5 7}$ (6), 100.

NajJari, M. R. \& Plesniak, M. W. 2018 Secondary flow vortical structures in a $180^{\circ}$ elastic curved vessel with torsion under steady and pulsatile inflow conditions. Phys. Rev. Fluids 3 (01).

Olson, D. E. \& SNyder, B. 1985 The upstream scale of flow development in curved circular pipes. J. Fluid Mech. 150, 139-158.

Plesniak, M. \& Bulusu, K. 2016 Morphology of secondary flows in a curved pipe with pulsatile inflow 138 (10).

Potter, M. C., Wiggert, D. C. \& Ramadan, B. H. 2016 Chapter 4: The integral forms of the fundamental laws. In Mechanics of Fluids. Nelson Education.

Ray, S., Ünsal, B., Durst, F., Ertunc, Ö. \& Bayoumi, O. A. 2005 Mass flow rate controlled fully developed laminar pulsating pipe flows. ASME .

Rindt, C. C. M., Van Steenhoven, A. A., Janssen, J. D. \& Vossers, G. 1991 Unsteady entrance flow in a $90^{\circ}$ curved tube. J. Fluid Mech. 226, 445-474.

Siggers, J. H. \& Waters, S. L. 2008 Unsteady flows in pipes with finite curvature. J. Fluid Mech. 600, 133-165.

Singh, M. P. 1974 Entry flow in a curved pipe. J. Fluid Mech. 65, 517-539.

Singh, P. J. \& MAdAVAn, N. K. 1987 Complete analysis and simulation of reciprocating pumps including system piping. In Proc. 4th Intl. Pump Users Symp.

Smith, F. T. 1975 Pulsatile flow in curved pipes. J. Fluid Mech. 71, 15-42.

Smith, F. T. 1976 Fluid flow into a curved pipe. Proc. R. Soc. Lond. A. 351 (1664), 71-87.

Sudo, K., Sumida, M. \& Yamane, R. 1992 Secondary motion of fully developed oscillatory flow in a curved pipe. J. Fluid Mech. 237, 189-208. 
Sumida, M. 2007 Pulsatile entrance flow in curved pipes: effect of various parameters. Exps. Fluids 43 (6), 949-958.

Sumida, M., Sudou, K. \& Wada, H. 1989 Pulsating flow in a curved pipe (secondary flow). JSME Int. J. Fluids Eng., Heat Trans., Pow., Comb., Ther. Prop. 32 (4), 523-531.

Takeshi, N., Yoshiniro, N., Sachiko, K. \& Kazuo, T. 1990 Developing oscillatory flow in a strongly curved tube. T. J. S. Mech. Eng. B. 56 (529), 2562-2569.

TAy, B. L. \& Thorpe, R. B. 2014 Hydrodynamic forces acting on pipe bends in gas-liquid slug flow. Chem. Eng. Res. Des. 92 (05), 812-825.

Thomson, J. 1877 On the origin of windings of rivers in alluvial plains, with remarks on the flow of water round bends in pipes. Proc. R. Soc. Lond. A. 25, 5-8.

Timite, B., Castelain, C. \& Peerhossain, H. 2010 Pulsatile viscous flow in a curved pipe: Effects of pulsation on the development of secondary flow. Intl. J. Heat Fluid Flow B. 31 (05), 879-896.

Tовак, M. \& Peake, D. J. 1982 Topology of three-dimensional separated flows. Ann. Rev. F. Mech. 14 (1), 61-85.

UCHIDA, S. 1956 The pulsating viscous flow superposed on the steady laminar motion of incompressible fluid in a circular pipe. Zeitschrift für angewandte Mathematik und Physik ZAMP 7 (5), 403-422.

Venudas, P. A. \& MAnu, K. V. 2018 Analytical solution of transient pipe flow for an arbitrary inflow. Proc. 7th Int. 45th N. Conf. Fluid Mech. Fluid Pow. IIT Bombay .

Vester, A. K., Örlü, R. \& Alfredsson, P. H. 2013 Vortical patterns in turbulent flow downstream a $90^{\circ}$ curved pipe at high womersley numbers. Intl. J. Heat Fluid Flow 44, 692-699.

Vester, A. K., Örlü, R. \& Alfredsson, P. H. 2015 Pulsatile turbulent flow in straight and curved pipes - interpretation and decomposition of hot-wire signals. Flow, Turb. Comb. $94(2), 305-321$.

Vester, A. K., Örlü, R. \& Alfredsson, P. H. 2016 Turbulent flows in curved pipes: recent advances in experiments, simulations and analysis. App. Mech. Rev. 68 (05).

Vetter, G. \& Schweinfurther, F. 1987 Pressure pulsations in the piping of reciprocating pumps. Chem. Eng. \& Tech. 10 (1), 262-271.

Wachel, J. C., Tison, J. D. \& Price, S. M. 1989 The effect of pulsations on cavitation in reciprocating pump systems. $A S M E$.

White, F. M. 2016 Chapter 3: Integral relations for a control volume. In Fluid mechanics. McGraw-Hill Education.

XiaO, F., Jing, J., Han, L., YAng, L. \& Wang, S. 2019 Modelling and analysis of impact forces acting on elbow in gas-liquid slug flow. Asia-Pacific J. Chem. Eng. 14 (02).

YAo, L. S. \& Berger, S. A. 1975 Entry flow in a curved pipe. J. Fluid Mech. 67, 177-196.

Yunus, A. C. 2017 Chapter 6: Momentum analysis of flow systems. In Fluid Mechanics: Fundamentals and applications. McGraw-Hill Education. 